Provided for non-commercial research and education use. Not for reproduction, distribution or commercial use.

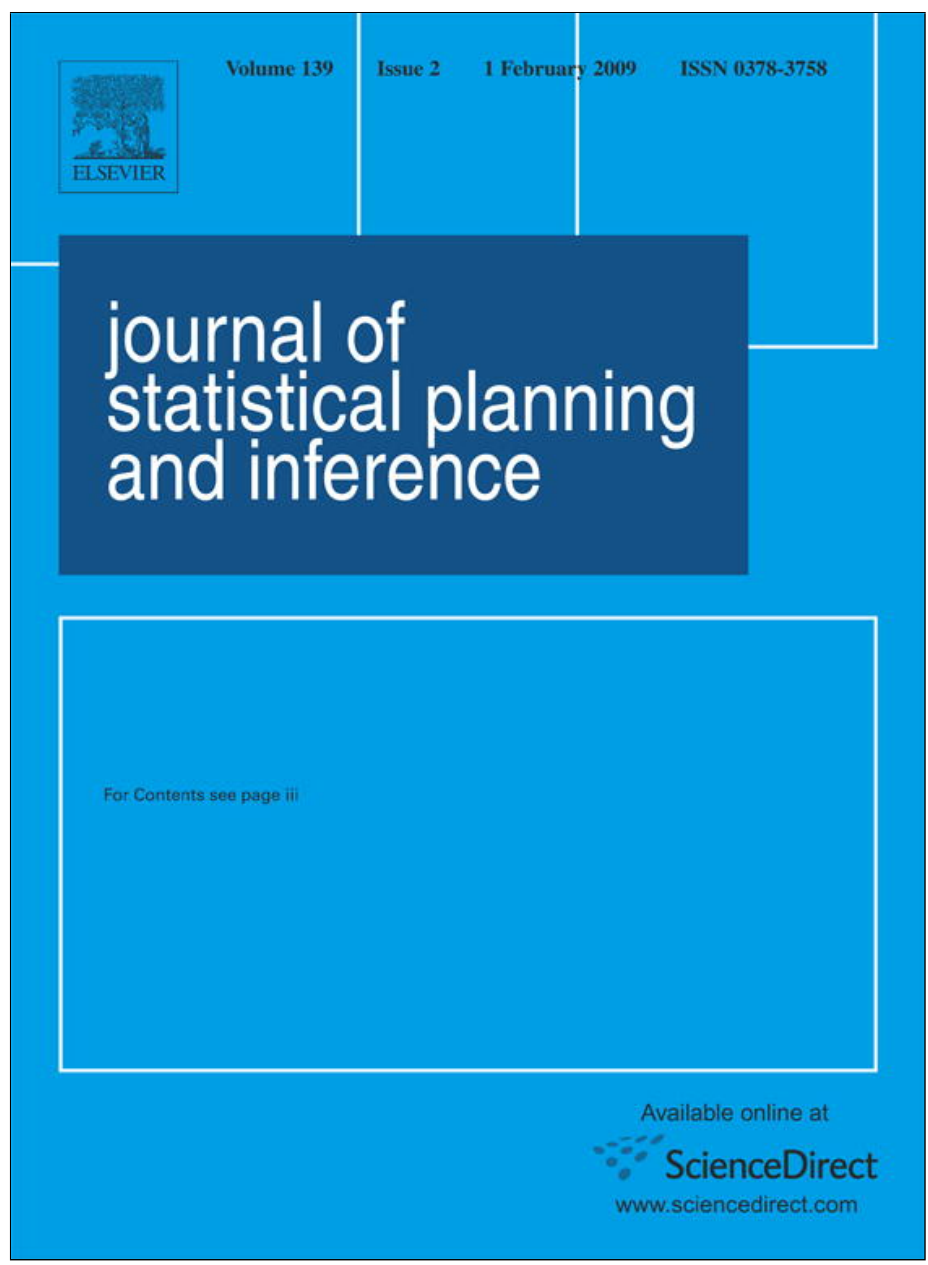

This article appeared in a journal published by Elsevier. The attached copy is furnished to the author for internal non-commercial research and education use, including for instruction at the authors institution and sharing with colleagues.

Other uses, including reproduction and distribution, or selling or licensing copies, or posting to personal, institutional or third party websites are prohibited.

In most cases authors are permitted to post their version of the article (e.g. in Word or Tex form) to their personal website or institutional repository. Authors requiring further information regarding Elsevier's archiving and manuscript policies are encouraged to visit:

http://www.elsevier.com/copyright 


\title{
Robust prediction and extrapolation designs for censored data
}

\author{
Xiaojian $\mathrm{Xu}$ \\ Department of Mathematics, Brock University, St. Catharines, Ontario, Canada L2S 3A1
}

\section{A R T I C L E I N F O}

\section{Article history:}

Received 17 May 2007

Received in revised form

19 December 2007

Accepted 9 May 2008

Available online 18 May 2008

\section{MSC:}

primary 62K05 62F35

secondary 62J12

Keywords:

Regression design

Maximum likelihood estimation

Unbiased design

Nonsmooth optimization

Accelerated life testing

Design implementation

\begin{abstract}
A B S T R A C T
In this paper we present the construction of robust designs for a possibly misspecified generalized linear regression model when the data are censored. The minimax designs and unbiased designs are found for maximum likelihood estimation in the context of both prediction and extrapolation problems. This paper extends preceding work of robust designs for complete data by incorporating censoring and maximum likelihood estimation. It also broadens former work of robust designs for censored data from others by considering both nonlinearity and much more arbitrary uncertainty in the fitted regression response and by dropping all restrictions on the structure of the regressors. Solutions are derived by a nonsmooth optimization technique analytically and given in full generality. A typical example in accelerated life testing is also demonstrated. We also investigate implementation schemes which are utilized to approximate a robust design having a density. Some exact designs are obtained using an optimal implementation scheme.
\end{abstract}

(c) 2008 Elsevier B.V. All rights reserved.

\section{Introduction}

We investigate the construction of designs for both prediction and extrapolation of a regression response incorporating censored data. Such designs are of interest in problems of life testing where there are two commonly used testing-time saving plans: censoring and acceleration. In this article, we will address both.

Generally speaking, tests yielding complete data take too long to run especially for those products having long life-spans. To save time, the testing results can be analyzed before all units fail. The data then consist of lifetime information on unfailed units, so-called censored data. Another purpose of censoring is that one can analyze the most recent test data while the test is still running.

When life testing runs at the stress levels within the range that the product would be normally used, the goal is prediction, namely the estimation of the mean response throughout the region of interest. For accelerated life testing (ALT), in which products are tested at higher than normal usage stress levels, the goal is extrapolation. For an extrapolation problem, if one is interested in estimating the mean response at a particular normal usage stress level which is lower than testing stress levels, we call it a one-point extrapolation problem; if one's interest falls into estimating that at certain range of normal usage stress levels, we call it a general extrapolation problem.

There is considerable literature regarding robust regression designs for a possibly misspecified linear response when observations are complete. For prediction problems, robust designs have been studied by Box and Draper (1959), Huber (1975), and Wiens (1992, 1998). For extrapolation problems, robust designs were investigated by Draper and Herzberg (1973), Huber (1975), Lawless (1984), Spruill (1984), Fang and Wiens (1999), and Wiens and Xu (2008a, b).

E-mail address: xxu@brocku.ca. 
For nonlinear regression problems without the consideration of model uncertainty, Atkinson and Haines (1996) and Ford et al. (1989) presented various static and sequential designs; for those considering robustness, Sinha and Wiens (2002) provided the construction of sequential designs which were robust against model uncertainty, and Wiens and Xu (2008a, b) discussed the construction of static designs which were robust against possibly misspecification in nonlinear models.

Recent work on robust designs with censored data in ALT are reported by Chaloner and Larntz (1992), Pascual and Montepiedra (2002, 2003), and most recently by Pascual (2006), and Zhang and Meeker (2006) who also provide a review on both Bayesian and non-Bayesian designs for ALT. These studies emphasize the robustness against misspecification on either the unknown model parameters or the underlying distribution and assume that the "true" model belongs to, or is distributed, with a known prior, onto a set of several known candidates. Ginebra and Sen (1998) investigated optimal designs, which are robust against possibly misspecified parameter values on which the optimal designs depend. The explicit designs obtained in those works are mostly under straight line or quadratic regression.

This present work focuses on the robustness against possible misspecification in regression functions that describe the behavior of mean responses in relation to the explanatory variables. Such misspecification generates a bias in the estimation of the mean response. We assume that the "true" model involves an unknown member of a certain contamination class but may not be the assumed one. This work broadens the previous work of robust designs with censoring from others by considering both nonlinearity and much more arbitrary uncertainty in the fitted regression response and by dropping all restrictions on the structure of regressors. It also extends previous work on robust designs for such model settings for complete data in Wiens and $\mathrm{Xu}(2008 \mathrm{a}, \mathrm{b})$ by incorporating censoring and maximum likelihood estimation.

The regression model considered in this study is similar to those in Wiens and Xu (2008a), however, the data investigated here are censored. To illustrate this treatment, the following outline is presented.

We consider a singly censored design with a specified censoring time for each stress level. The underlying distribution is assumed to be normal. For the lifetime, the underlying distribution is usually considered to be normal after the observations are transformed. For example, suppose some product's lifetime is possibly a lognormal distribution. In such case, we take the logarithm of all observations and censoring times, then carry out the regression method on the data after such transformations. Let $Y$ be the random variable, for instance, (transformed) lifetime of the product or material, $\zeta$ be the (correspondingly transformed) censoring time which is constant for a fixed stress level, and let $\mathbf{x}$ represent the stress level and be a $q$-dimensional vector belonging to a bounded design space $S$.

We consider

$$
Y\left(\mathbf{x}_{i}\right)=E\left(Y \mid \mathbf{x}_{i}\right)+\varepsilon_{i},
$$

with design points $\mathbf{x}_{1}, \mathbf{x}_{2}, \ldots, \mathbf{x}_{n}$ freely chosen from $S$, where the $\varepsilon_{i}$ 's are uncorrelated and identically distributed with a normal distribution and a common variance $\sigma^{2}$. In this paper, we mainly address the construction of optimal robust design for homoscedasticity. We note that the analytic form for the optimal robust design with respect to heteroscedasticity, i.e. $\sigma^{2}=\sigma^{2}(\mathbf{x})$, can also be found with very similar derivations to that for the homoscedasticity case. However, this form involves more coefficients to be determined by the last step of numerical loss minimization procedure. See Remark 1 in Section 3.1.

We assume that the mean response is regarded as being an only approximately known function of unknown parameters, and a linear function of a given $p \times 1$ regressor vector $\mathbf{z}^{\mathrm{T}}(\mathbf{x})$, depending on $q$-dimentional vector $\mathbf{x}$ of explanatory variables, $x_{1}, x_{2}, \ldots, x_{q}$ :

$$
E(Y \mid \mathbf{x}) \approx h\left(\mathbf{z}^{\mathrm{T}}(\mathbf{x}) \boldsymbol{\theta}_{0}\right)
$$

The function $h$ is strictly monotonic with a bounded second derivative. We assume $\left\|\mathbf{z}^{\mathrm{T}}(\mathbf{x})\right\|$ is bounded on $S$. The "correct" vector $\boldsymbol{\theta}_{0}$ of regression parameters may be defined by

$$
\boldsymbol{\theta}_{0}=\arg \min _{\mathbf{t}} \int_{S}\left[E(Y \mid \mathbf{x})-h\left(\mathbf{z}^{\mathrm{T}}(\mathbf{x}) \mathbf{t}\right)\right]^{2} \mathrm{~d} \mathbf{x}
$$

Then after introducing $f_{n}(\mathbf{x})=\sqrt{n}\left[E(Y \mid \mathbf{x})-h\left(\mathbf{z}^{\mathrm{T}}(\mathbf{x}) \boldsymbol{\theta}_{0}\right)\right]$, we obtain

$$
E(Y \mid \mathbf{x})=h\left(\mathbf{z}^{\mathrm{T}}(\mathbf{x}) \boldsymbol{\theta}_{0}\right)+n^{-1 / 2} f_{n}(\mathbf{x}) .
$$

Whenever it is clear from the context, we drop the subscript $n$ on $f_{n}$. The contaminant $f$ is unknown but relatively "small". Such misspecification may be generated by a transformation of the data for the purpose of enhancing normality. It may also be "viewed as arising from imprecision in the specification of $h$, or it can arise from a misspecified linear term and a two-term Taylor expansion" as discussed in Wiens and $\mathrm{Xu}(2008 \mathrm{~b})$.

We let $\tilde{\mathbf{z}}(\mathbf{x})=\left(\mathrm{d} h /\left.\mathrm{d} \mu\right|_{\boldsymbol{\theta}=\boldsymbol{\theta}_{0}}\right) \mathbf{z}(\mathbf{x})$ with $\mu=\mathbf{z}^{\mathrm{T}}(\mathbf{x}) \boldsymbol{\theta}$. By the definition of $\boldsymbol{\theta}_{0}$, we have

$$
\int_{S} \tilde{\mathbf{z}}^{\mathrm{T}}(\mathbf{x}) f(\mathbf{x}) \mathrm{d} \mathbf{x}=0
$$

To ensure that $\boldsymbol{\theta}_{0}$ is well-defined, we need both (2) and $\int_{S} \tilde{\mathbf{z}}(\mathbf{x}) \tilde{\mathbf{z}}^{\mathrm{T}}(\mathbf{x})$ d $\mathbf{x}$ being nonsingular which holds from Assumption (A) in Section 2. Let $\hat{\boldsymbol{\theta}}$ be the maximum likelihood estimator (MLE) of $\boldsymbol{\theta}_{0}$ obtained from the censored data. We define the loss functions 
for the following cases:

(1) For prediction problems, where we attempt to estimate the mean response $E(Y \mid \mathbf{x})$ on the entire design space $S$, let

$$
I_{1}=n \int_{S} E\left[h\left(\mathbf{z}^{\mathrm{T}}(\mathbf{x}) \hat{\boldsymbol{\theta}}\right)-E(Y \mid \mathbf{x})\right]^{2} \mathrm{~d} \mathbf{x}
$$

(2) For extrapolation problems, we take

$$
I_{2}=n \int_{T} E\left[h\left(\mathbf{z}^{\mathrm{T}}(\mathbf{x}) \hat{\boldsymbol{\theta}}\right)-E(Y \mid \mathbf{x})\right]^{2} \omega(\mathrm{d} \mathbf{x})
$$

where $\omega\left\{\mathbf{x}_{0}\right\}=1$ in the case of one-point extrapolation when we estimate $E\left(Y \mid \mathbf{x}_{0}\right)$, at $\mathbf{x}_{0} \notin S$, by $\hat{Y}\left(\mathbf{x}_{0}\right)=h\left(\mathbf{z}^{\mathrm{T}}\left(\mathbf{x}_{0}\right) \hat{\boldsymbol{\theta}}\right)$; and $\omega$ is a Lebesgue measure in the case of general extrapolation with extrapolation region $T$ assuming $\omega(T)>0$, and $T \cap S=\phi$, where we intend to extrapolate $E(Y \mid \mathbf{x})$ to the entire region $T$.

These loss functions depend on the design measure $\xi=n^{-1} \sum_{i=1}^{n} I\left(\mathbf{x}=\mathbf{x}_{i}\right)$, where $I$ is the indicator function, as well as on $f$. The following problems will be addressed in this paper sequentially:

P1: Determine designs to minimize the maximum value of $I_{1}$ over $f$.

P2: Determine designs to minimize the maximum value of $I_{2}$ over $f$ for one-point extrapolation.

P3: Determine designs to minimize the maximum value of $I_{2}$ over $f$ for general extrapolation.

P4, P5, and P6: Determine unbiased designs in the context of prediction, one-point extrapolation, and general extrapolation, respectively.

We assume that the contaminant $f$ involved in P1-P6 varies within certain specified contamination classes.

The rest of this paper is arranged as follows: Some mathematical preliminaries and notation are detailed in Section 2; the designs for P1-P3 are presented in Section 3; the designs for P4-P6 are delineated in Section 4; the computation of the resulting designs has been demonstrated using a typical ALT example in Section 5; in Section 6, the implementation schemes are discussed and some implementable designs are obtained. A list of the symbols used and derivations of all theorems in this article are provided in the appendixes.

\section{Preliminaries and notation}

For any observation $y(\mathbf{x})$ at stress level $\mathbf{x}$, we define an indicator function, $c=c(y \mid \mathbf{x})$ in terms of the censoring time $\zeta(\mathbf{x})$ by letting it be 1 when an failure occurs and 0 when observation is censored, i.e.

$$
c(y \mid \mathbf{x})= \begin{cases}1 & \text { when } y(\mathbf{x}) \leqslant \zeta(\mathbf{x}) \\ 0 & \text { when } y(\mathbf{x})>\zeta(\mathbf{x})\end{cases}
$$

Let $\phi$ and $\Phi$ be the standard normal density and cumulative distribution function, respectively. Under the fitted regression model $E(Y \mid \mathbf{x})=h\left(\mathbf{z}^{\mathrm{T}}(\mathbf{x}) \boldsymbol{\theta}\right)$, the log likelihood of the $i$ th individual observation $\left(y_{i}, c_{i}\right)$ at stress level $\mathbf{x}_{i}$ is

$$
l_{i}=c_{i}\left\{-\ln (\sigma)-\frac{1}{2} \ln (2 \pi)-\frac{1}{2}\left(\frac{y_{i}-h\left(\mathbf{z}^{\mathrm{T}}\left(\mathbf{x}_{i}\right) \boldsymbol{\theta}\right)}{\sigma}\right)^{2}\right\}+\left(1-c_{i}\right)\left\{\ln \left[1-\Phi\left(\frac{\zeta\left(\mathbf{x}_{i}\right)-h\left(\mathbf{z}^{\mathrm{T}}\left(\mathbf{x}_{i}\right) \boldsymbol{\theta}\right)}{\sigma}\right)\right]\right\} .
$$

Denote the standardized observation and censoring time at a specified stress level $\mathbf{x}$ by $w(\mathbf{x})$ and $\tau(\mathbf{x})$, i.e. $w(\mathbf{x})=\left(y(\mathbf{x})-h\left(\mathbf{z}^{\mathrm{T}}(\mathbf{x}) \boldsymbol{\theta}_{0}\right)\right) / \sigma$ and $\tau(\mathbf{x})=\left(\zeta(\mathbf{x})-h\left(\mathbf{z}^{\mathrm{T}}(\mathbf{x}) \boldsymbol{\theta}_{0}\right)\right) / \sigma$. Then we have

$$
\left.\frac{\partial l_{i}}{\partial \boldsymbol{\theta}}\right|_{\boldsymbol{\theta}=\boldsymbol{\theta}_{0}}=\frac{\tilde{\mathbf{z}}^{\mathrm{T}}\left(\mathbf{x}_{i}\right)}{\sigma}\left\{c_{i} w_{i}+\left(1-c_{i}\right) \frac{\phi\left(\tau_{i}\right)}{1-\Phi\left(\tau_{i}\right)}\right\},
$$

and

$$
\left.\frac{\partial^{2} l_{i}}{\partial \boldsymbol{\theta} \partial \boldsymbol{\theta}^{\mathrm{T}}}\right|_{\boldsymbol{\theta}=\boldsymbol{\theta}_{0}}=-\frac{\tilde{\mathbf{z}}\left(\mathbf{x}_{i}\right) \tilde{\mathbf{z}}^{\mathrm{T}}\left(\mathbf{x}_{i}\right)}{\sigma^{2}}\left\{c_{i}+\left(1-c_{i}\right)\left[\left(\frac{\phi\left(\tau_{i}\right)}{1-\Phi\left(\tau_{i}\right)}\right)^{2}-\frac{\tau_{i} \phi\left(\tau_{i}\right)}{1-\Phi\left(\tau_{i}\right)}\right]\right\}
$$

where $\tau_{i}=\tau\left(\mathbf{x}_{i}\right), c_{i}=c\left(\mathbf{x}_{i}\right)$ and $w_{i}=w\left(\mathbf{x}_{i}\right)$. Both expressions above involve two random variables: $c_{i}$ and $c_{i} w_{i}$. The following derives the expectations of, variances of, and covariance between these two variables based on the "true" model. 
We notice that $c_{i}$ and $w_{i}$ have the following distributions:

$$
\begin{aligned}
& c_{i} \sim \operatorname{bin}\left(1, P_{i}\right) \quad \text { with } P_{i}=P\left(c_{i}=1\right), \\
& w_{i} \sim \mathrm{N}\left(\frac{f\left(\mathbf{x}_{i}\right)}{\sqrt{n} \sigma}, 1\right) .
\end{aligned}
$$

and

$$
c_{i} w_{i}= \begin{cases}w_{i} & \text { when } w_{i} \leqslant \tau_{i} \\ 0 & \text { when } w_{i}>\tau_{i}\end{cases}
$$

According to (1), we obtain

$$
\begin{aligned}
E\left(c_{i}\right) & =\Phi\left(\tau_{i}\right)-\phi\left(\tau_{i}\right) \frac{f\left(\mathbf{x}_{i}\right)}{\sqrt{n} \sigma}+o\left(\frac{f\left(\mathbf{x}_{i}\right)}{\sqrt{n}}\right), \\
E\left(c_{i} w_{i}\right) & =-\phi\left(\tau_{i}\right)+\left[\Phi\left(\tau_{i}\right)-\tau_{i} \phi\left(\tau_{i}\right)\right] \frac{f\left(\mathbf{x}_{i}\right)}{\sqrt{n} \sigma}+o\left(\frac{f\left(\mathbf{x}_{i}\right)}{\sqrt{n}}\right), \\
\operatorname{Var}\left(c_{i}\right) & =\Phi\left(\tau_{i}\right)-\Phi^{2}\left(\tau_{i}\right)+\phi\left(\tau_{i}\right)\left[2 \Phi\left(\tau_{i}\right)-1\right] \frac{f\left(\mathbf{x}_{i}\right)}{\sqrt{n} \sigma}+o\left(\frac{f\left(\mathbf{x}_{i}\right)}{\sqrt{n}}\right), \\
\operatorname{Var}\left(c_{i} w_{i}\right) & =\left[\Phi\left(\tau_{i}\right)-\tau_{i} \phi\left(\tau_{i}\right)-\phi^{2}\left(\tau_{i}\right)\right]+\phi\left(\tau_{i}\right)\left[2 \Phi\left(\tau_{i}\right)-2 \tau_{i} \phi\left(\tau_{i}\right)-\tau_{i}^{2}+2\right] \frac{f\left(\mathbf{x}_{i}\right)}{\sqrt{n} \sigma}+o\left(\frac{f\left(\mathbf{x}_{i}\right)}{\sqrt{n}}\right), \\
\operatorname{Cov}\left(c_{i}, c_{i} w_{i}\right) & =-\phi\left(\tau_{i}\right)\left[1-\Phi\left(\tau_{i}\right)\right]-\left\{\phi^{2}\left(\tau_{i}\right)+\left[1-\Phi\left(\tau_{i}\right)\right]\left[\tau_{i} \phi\left(\tau_{i}\right)-\Phi\left(\tau_{i}\right)\right]\right\} \frac{f\left(\mathbf{x}_{i}\right)}{\sqrt{n} \sigma}+o\left(\frac{f\left(\mathbf{x}_{i}\right)}{\sqrt{n}}\right) .
\end{aligned}
$$

Hence,

$$
\begin{aligned}
& E\left(\left.\frac{\partial l_{i}}{\partial \boldsymbol{\theta}}\right|_{\boldsymbol{\theta}=\boldsymbol{\theta}_{0}}\right)=a\left(\tau_{i}\right) \frac{f\left(\mathbf{x}_{i}\right) \tilde{\mathbf{z}}^{\mathrm{T}}\left(\mathbf{x}_{i}\right)}{\sqrt{n} \sigma^{2}}+\mathrm{o}\left(\frac{f\left(\mathbf{x}_{i}\right)}{\sqrt{n}}\right), \\
& \left.E\left(-\left.\frac{\partial^{2} l_{i}}{\partial \boldsymbol{\theta} \partial \boldsymbol{\theta}^{\mathrm{T}}}\right|_{\boldsymbol{\theta}=\boldsymbol{\theta}_{0}}\right)=\frac{\tilde{\mathbf{z}}\left(\mathbf{x}_{i}\right) \tilde{\mathbf{z}}^{\mathrm{T}}\left(\mathbf{x}_{i}\right)}{\sigma^{2}}\left\{a\left(\tau_{i}\right)+\left(b\left(\tau_{i}\right)+a\left(\tau_{i}\right)\left[\left(\frac{\mathrm{d}^{2} h}{\mathrm{~d} \mu^{2}}\right)\left(\frac{\mathrm{d} h}{\mathrm{~d} \mu}\right)^{-2}\right]\right]_{\boldsymbol{\theta}=\boldsymbol{\theta}_{0}}\right) \frac{f\left(\mathbf{x}_{i}\right)}{\sqrt{n} \sigma}\right\}+\mathrm{o}\left(\frac{f\left(\mathbf{x}_{i}\right)}{\sqrt{n}}\right),
\end{aligned}
$$

and

$$
\operatorname{Cov}\left(\left.\frac{\partial l_{i}}{\partial \boldsymbol{\theta}}\right|_{\boldsymbol{\theta}=\boldsymbol{\theta}_{0}}\right)=\frac{\tilde{\mathbf{z}}\left(\mathbf{x}_{i}\right) \tilde{\mathbf{z}}^{\mathrm{T}}\left(\mathbf{x}_{i}\right)}{\sigma^{2}}\left\{a\left(\tau_{i}\right)+\left[2 \phi\left(\tau_{i}\right)-\tau_{i}^{2} \phi\left(\tau_{i}\right)+\frac{\phi^{3}\left(\tau_{i}\right)}{\left\{1-\Phi\left(\tau_{i}\right)\right\}^{2}}\right] \frac{f\left(\mathbf{x}_{i}\right)}{\sqrt{n} \sigma}\right\}+\mathrm{o}\left(\frac{f\left(\mathbf{x}_{i}\right)}{\sqrt{n}}\right),
$$

where $a(\tau)=\Phi(\tau)-\tau \phi(\tau)+\phi^{2}(\tau) /(1-\Phi(\tau))$ and $b(\tau)=\phi^{3}(\tau) /\left(\{1-\Phi(\tau)\}^{2}\right)-\tau \phi^{2}(\tau) /(1-\Phi(\tau))-\phi(\tau)$. We note that $a(\tau)$ is a function of $\mathbf{x}, \boldsymbol{\theta}_{0}$, and $\sigma$, but the dependency on $\mathbf{x}, \boldsymbol{\theta}_{0}$, and $\sigma$ is only through $\tau$. For readability, we use $a_{\tau}(\mathbf{x})$ when we emphasize its dependence on $\mathbf{x}$ with

$$
a_{\tau}(\mathbf{x})=\Phi\left(\frac{\zeta(\mathbf{x})-h\left(\mathbf{z}^{\mathrm{T}}(\mathbf{x}) \boldsymbol{\theta}_{0}\right)}{\sigma}\right)+\frac{\phi^{2}\left(\left(\zeta(\mathbf{x})-h\left(\mathbf{z}^{\mathrm{T}}(\mathbf{x}) \boldsymbol{\theta}_{0}\right)\right) / \sigma\right)}{1-\Phi\left(\left(\zeta(\mathbf{x})-h\left(\mathbf{z}^{\mathrm{T}}(\mathbf{x}) \boldsymbol{\theta}_{0}\right)\right) / \sigma\right)}-\frac{\zeta(\mathbf{x})-h\left(\mathbf{z}^{\mathrm{T}}(\mathbf{x}) \boldsymbol{\theta}_{0}\right)}{\sigma} \phi\left(\frac{\zeta(\mathbf{x})-h\left(\mathbf{z}^{\mathrm{T}}(\mathbf{x}) \boldsymbol{\theta}_{0}\right)}{\sigma}\right) .
$$

To avoid trivialities and to make sure the nonsingularity of a number of relevant matrices, we assume that the design space $S$ and extrapolation space $T$ satisfy

(A) For each vector $\mathbf{v} \neq \mathbf{0}$, the set $\left\{\mathbf{x} \in S \cup T: \mathbf{v}^{\mathrm{T}} \tilde{\mathbf{z}}(\mathbf{x})=\mathbf{0}\right\}$ has Lebesgue measure zero.

We assume

$$
\int_{S} f^{2}(\mathbf{x}) \mathrm{d} \mathbf{x} \leqslant \eta_{S}^{2}<\infty
$$

for a positive constant $\eta_{S}$, and also define the following matrices and vectors:

$$
\begin{array}{ll}
\mathbf{A}_{S}=\int_{S} \tilde{\mathbf{z}}(\mathbf{x}) \tilde{\mathbf{z}}^{\mathrm{T}}(\mathbf{x}) \mathrm{d} \mathbf{x}, & \mathbf{A}_{0}=\tilde{\mathbf{z}}\left(\mathbf{x}_{0}\right) \tilde{\mathbf{z}}^{\mathrm{T}}\left(\mathbf{x}_{0}\right), \\
\mathbf{A}_{T}=\int_{T} \tilde{\mathbf{z}}(\mathbf{x}) \tilde{\mathbf{z}}^{\mathrm{T}}(\mathbf{x}) \mathrm{d} \mathbf{x}, & \mathbf{B}=\int_{S} a(\mathbf{x}) \tilde{\mathbf{z}}(\mathbf{x}) \tilde{\mathbf{z}}^{\mathrm{T}}(\mathbf{x}) \xi(\mathrm{d} \mathbf{x}), \\
\mathbf{H}_{S}=\mathbf{B}^{-1} \mathbf{A}_{S} \mathbf{B}^{-1}, & \mathbf{H}_{0}=\mathbf{B}^{-1} \mathbf{A}_{0} \mathbf{B}^{-1}, \\
\mathbf{H}_{T}=\mathbf{B}^{-1} \mathbf{A}_{T} \mathbf{B}^{-1}, & \mathbf{b}_{f, S}=\int_{S} a(\mathbf{x}) \tilde{\mathbf{z}}(\mathbf{x}) f(\mathbf{x}) \xi(\mathrm{d} \mathbf{x}), \\
\mathbf{b}_{f, T}=\int_{T} \tilde{\mathbf{z}}(\mathbf{x}) f(\mathbf{x}) \mathrm{d} \mathbf{x} . &
\end{array}
$$

It follows from (A) that $\mathbf{A}_{S}, \mathbf{A}_{T}$ are nonsingular and that $\mathbf{B}$ is also nonsingular whenever $\xi$ does not place mass on sets of Lebesgue measure zero. This requirement turns out to be necessary since $\xi$ has to be absolutely continuous due to (3) as discussed later in this section. 
By virtue of our assumption on $f, \mathbf{z}$ and $h$ and the definition of $\mathbf{B}$ and $\mathbf{b}_{f, S}$, we obtain the following results. The asymptotic information matrix of $\theta_{0}$ is

$$
\begin{aligned}
\mathbf{I}\left(\boldsymbol{\theta}_{0}\right) & =\lim _{n \rightarrow \infty} \frac{1}{n} E\left(-\left.\sum_{i=1}^{n} \frac{\partial^{2} l_{i}}{\partial \boldsymbol{\theta} \partial \boldsymbol{\theta}^{\mathrm{T}}}\right|_{\boldsymbol{\theta}=\boldsymbol{\theta}_{0}}\right) \\
& =\frac{1}{\sigma^{2}} \int_{S} a(\mathbf{x}) \tilde{\mathbf{z}}(\mathbf{x}) \tilde{\mathbf{z}}^{\mathrm{T}}(\mathbf{x}) \xi(\mathrm{d} \mathbf{x}) \\
& =\frac{1}{\sigma^{2}} \mathbf{B} .
\end{aligned}
$$

The asymptotic expectation of the score function evaluated at $\boldsymbol{\theta}_{0}$ is

$$
\begin{aligned}
\tilde{\mathbf{b}}_{f}\left(\boldsymbol{\theta}_{0}\right) & =\frac{1}{\sqrt{n}} \lim _{n \rightarrow \infty} \frac{1}{n} E\left(\left.\sqrt{n} \sum_{i=1}^{n} \frac{\partial l_{i}}{\partial \boldsymbol{\theta}}\right|_{\boldsymbol{\theta}=\boldsymbol{\theta}_{0}}\right) \\
& =\frac{1}{\sqrt{n} \sigma^{2}} \int_{S} a(\mathbf{x}) \tilde{\mathbf{z}}(\mathbf{x}) f(\mathbf{x}) \xi(\mathrm{d} \mathbf{x}) \\
& =\frac{1}{\sqrt{n} \sigma^{2}} \mathbf{b}_{f, S} .
\end{aligned}
$$

And the asymptotic variance-covariance matrix of the score function evaluated at $\theta_{0}$ is

$$
\begin{aligned}
\mathbf{C}\left(\boldsymbol{\theta}_{0}\right) & =\lim _{n \rightarrow \infty} \frac{1}{n} \sum_{i=1}^{n} \operatorname{Cov}\left(\left.\frac{\partial l_{i}}{\partial \boldsymbol{\theta}}\right|_{\boldsymbol{\theta}=\boldsymbol{\theta}_{0}}\right) \\
& =\frac{1}{\sigma^{2}} \int_{S} a(\mathbf{x}) \tilde{\mathbf{z}}(\mathbf{x}) \tilde{\mathbf{z}}^{\mathrm{T}}(\mathbf{x}) \xi(\mathrm{d} \mathbf{x}) \\
& =\frac{1}{\sigma^{2}} \mathbf{B} .
\end{aligned}
$$

Since the maximum likelihood estimate $\hat{\theta}$ is a root of the score function which can be expanded around $\theta_{0}$ as

$$
\frac{1}{n}\left(\left.\sum_{i=1}^{n} \frac{\partial l_{i}}{\partial \boldsymbol{\theta}}\right|_{\boldsymbol{\theta}=\boldsymbol{\theta}_{0}}\right)+\frac{1}{n}\left(-\left.\sum_{i=1}^{n} \frac{\partial^{2} l_{i}}{\partial \boldsymbol{\theta} \partial \boldsymbol{\theta}^{\mathrm{T}}}\right|_{\boldsymbol{\theta}=\boldsymbol{\theta}_{0}}\right)\left(\hat{\boldsymbol{\theta}}-\boldsymbol{\theta}_{0}\right)+\mathrm{O}\left(\left(\hat{\boldsymbol{\theta}}-\boldsymbol{\theta}_{0}\right)^{\mathrm{T}}\left(\hat{\boldsymbol{\theta}}-\boldsymbol{\theta}_{0}\right)\right),
$$

we have

$$
\hat{\boldsymbol{\theta}}-\boldsymbol{\theta}_{0} \approx \frac{1}{n}\left(\left.\sum_{i=1}^{n} \frac{\partial l_{i}}{\partial \boldsymbol{\theta}}\right|_{\boldsymbol{\theta}=\boldsymbol{\theta}_{0}}\right) \mathbf{I}^{-1}\left(\boldsymbol{\theta}_{0}\right)
$$

Consequently, the asymptotic distribution of $\sqrt{n}\left(\hat{\boldsymbol{\theta}}-\boldsymbol{\theta}_{0}\right)$ is then

$$
\sqrt{n}\left(\hat{\boldsymbol{\theta}}-\boldsymbol{\theta}_{0}\right) \sim \operatorname{AN}\left(\mathbf{B}^{-1}\left(\boldsymbol{\theta}_{0}\right) \mathbf{b}_{f, S}\left(\boldsymbol{\theta}_{0}\right), \sigma^{2} \mathbf{B}^{-1}\left(\boldsymbol{\theta}_{0}\right)\right) .
$$

We denote

$$
\begin{aligned}
& \mathscr{F}_{1}=\left\{f \mid \int_{S} \tilde{\mathbf{z}}(\mathbf{x}) f(\mathbf{x}) \mathrm{d} \mathbf{x}=0, \quad \int_{S} f^{2}(\mathbf{x}) \mathrm{d} \mathbf{x} \leqslant \eta_{S}^{2}<\infty\right\}, \\
& \mathscr{F}_{2}=\left\{f\left|\int_{S} \tilde{\mathbf{z}}(\mathbf{x}) f(\mathbf{x}) \mathrm{d} \mathbf{x}=0, \quad \int_{S} f^{2}(\mathbf{x}) \mathrm{d} \mathbf{x} \leqslant \eta_{S}^{2}<\infty,\right| f\left(\mathbf{x}_{0}\right) \mid \leqslant \eta_{0}<\infty\right\},
\end{aligned}
$$

and

$$
\mathscr{F}_{3}=\left\{f \mid \int_{S} \tilde{\mathbf{z}}(\mathbf{x}) f(\mathbf{x}) \mathrm{d} \mathbf{x}=0, \quad \int_{S} f^{2}(\mathbf{x}) \mathrm{d} \mathbf{x} \leqslant \eta_{S}^{2}<\infty, \quad \int_{T} f^{2}(\mathbf{x}) \mathrm{d} \mathbf{x} \leqslant \eta_{T}^{2}<\infty\right\}
$$

for positive constants $\eta_{0}$ and $\eta_{T}$. For the regression model (1), we assume that the contamination function $f(\mathbf{x})$ is an unknown member of one of the classes above. In fact, since the contamination classes above are so full, $\xi$ has to have a density in order to guarantee $\sup _{f \in \mathscr{F}_{j}} I_{i}$, with $(i, j)=(1,1),(2,2)$, or $(2,3)$, is finite. This can be established by modifying the proof of Lemma 1 of Wiens (1992). To implement the optimal design $\xi$ in practice, however, it must be approximated by a discrete design. Approximation methods are discussed in Section 6. 
Let $k(\mathbf{x})$ be the density of $\xi(\mathbf{x})$, then we have

$$
\begin{aligned}
& \mathbf{B}=\int_{S} a(\mathbf{x}) k(\mathbf{x}) \tilde{\mathbf{z}}(\mathbf{x}) \tilde{\mathbf{z}}^{\mathrm{T}}(\mathbf{x}) \mathrm{d} \mathbf{x}, \\
& \mathbf{b}_{f, S}=\int_{S} a(\mathbf{x}) k(\mathbf{x}) \tilde{\mathbf{z}}(\mathbf{x}) f(\mathbf{x}) \mathrm{d} \mathbf{x} .
\end{aligned}
$$

We also define $\mathbf{K}=\int_{S} a^{2}(\mathbf{x}) k^{2}(\mathbf{x}) \tilde{\mathbf{z}}(\mathbf{x}) \tilde{\mathbf{z}}^{\mathrm{T}}(\mathbf{x}) \mathrm{d} \mathbf{x}$ and $\mathbf{G}=\mathbf{K}-\mathbf{B} \mathbf{A}_{S}^{-1} \mathbf{B}$. We note that

(1) $\mathbf{G}$ is positive semidefinite since, for any vector $\mathbf{c}$,

$$
\left.\mathbf{c}^{\mathrm{T}} \mathbf{G} \mathbf{c}=\int_{S}\left\{\mathbf{c}^{\mathrm{T}}\left[k(\mathbf{x}) a(\mathbf{x}) \mathbf{I}-\mathbf{B} \mathbf{A}_{S}^{-1}\right) \tilde{\mathbf{z}}(\mathbf{x})\right]\right\}^{2} \mathrm{~d} \mathbf{x} \geqslant 0 ;
$$

(2) $a$ is a nonnegative function since $a(\tau)[1-\Phi(\tau)]=[\Phi(\tau)-\tau \phi(\tau)][1-\Phi(\tau)]+\phi^{2}(\tau)$ with $\Phi(\tau)-\tau \phi(\tau)>0$.

\section{Minimax designs for censored data}

In this section, we investigate the optimal designs that minimize the maximum value of the loss, over $f$, in the following three cases:

(P1) prediction problems with $f \in \mathscr{F}_{1}$;

(P2) one-point extrapolation problems with $f \in \mathscr{F}_{2}$; and

(P3) general extrapolation problems with $f \in \mathscr{F}_{3}$.

\subsection{Minimax designs for prediction: solutions to P1}

The loss function for Problem P1 is

$$
\begin{aligned}
I_{1} & =n \int_{S} E\left[h\left(\mathbf{z}^{\mathrm{T}}(\mathbf{x}) \hat{\boldsymbol{\theta}}\right)-h\left(\mathbf{z}^{\mathrm{T}}(\mathbf{x}) \boldsymbol{\theta}_{0}\right)-n^{-1 / 2} f(\mathbf{x})\right]^{2} \mathrm{~d} \mathbf{x} \\
& =\mathbf{b}_{f, S}^{\mathrm{T}} \mathbf{H}_{S}^{-1} \mathbf{b}_{f, S}+\sigma \operatorname{tr}\left(\mathbf{A}_{S} \mathbf{B}^{-1}\right)+\int_{S} f^{2}(\mathbf{x}) \mathrm{d} \mathbf{x} .
\end{aligned}
$$

Let $v:=\sigma^{2} / \eta_{S}^{2}$ represent the relative importance of variance versus bias. We define $\lambda_{k}^{(1)}$ to be the largest solution to $\left|\mathbf{G}-\lambda \mathbf{H}_{S}\right|=0$ and $\mathbf{c}_{1}$ to be any vector satisfying $\left(\mathbf{G H}_{S}^{-1} \mathbf{G}-\lambda_{k}^{(1)} \mathbf{G}\right) \mathbf{c}=0$, and

$$
\mathbf{c}^{\mathrm{T}} \mathbf{G} \mathbf{c}=1
$$

Given fixed $k(\mathbf{x})$, the "max" part of the minimax solution is presented in Theorem 1 .

Theorem 1. The maximum of $I_{1}$ is

$$
\sup _{f \in \mathscr{F}_{1}} I_{1}(f, \xi)=\eta_{S}^{2}\left[\lambda_{k}^{(1)}+1+v \operatorname{tr}\left(\mathbf{A}_{S} \mathbf{B}^{-1}\right)\right]
$$

attained at

$$
f_{k}(\mathbf{x})=\eta_{S} \tilde{\mathbf{z}}^{\mathrm{T}}(\mathbf{x})\left\{a(\mathbf{x}) k(\mathbf{x}) \mathbf{I}-\mathbf{A}_{S}^{-1} \mathbf{B}\right\} \mathbf{c}_{1} .
$$

Problem P1 has become one of finding a density $k(\mathbf{x})$ that minimizes (5). The following theorem provides the analytical form of such minimax design density.

Theorem 2. The design density $k(\mathbf{x})$ minimizing (5) for prediction is of the form

$$
k(\mathbf{x})=\frac{\left[a(\mathbf{x}) \tilde{\mathbf{z}}^{\mathrm{T}}(\mathbf{x}) \mathbf{P} \tilde{\mathbf{z}}(\mathbf{x})+d\right]^{+}}{a^{2}(\mathbf{x}) \tilde{\mathbf{z}}^{\mathrm{T}}(\mathbf{x}) \mathbf{Q} \tilde{\mathbf{z}}(\mathbf{x})},
$$

where $(w)^{+}=\max (w, 0)$, for some constant symmetric matrix $\mathbf{P}$, a positive semi-definite matrix $\mathbf{Q}$ and a constant $d$ that minimize (5) and satisfy $\int_{S} k(\mathbf{x}) \mathrm{d} \mathbf{x}=1$. 
Remark 1. For the case of unequal variances $\sigma^{2}(\mathbf{x})$, the optimal design density $k(\mathbf{x})$ for prediction is of the form

$$
k(\mathbf{x})=\frac{\left[a(\mathbf{x}) \sigma^{2}(\mathbf{x}) \tilde{\mathbf{z}}^{\mathrm{T}}(\mathbf{x}) \mathbf{P} \tilde{\mathbf{z}}(\mathbf{x})-a(\mathbf{x}) \tilde{\mathbf{z}}^{\mathrm{T}}(\mathbf{x}) \mathbf{T} \tilde{\mathbf{z}}(\mathbf{x})+d\right]^{+}}{a^{2}(\mathbf{x}) \tilde{\mathbf{z}}^{\mathrm{T}}(\mathbf{x}) \mathbf{Q} \tilde{\mathbf{z}}(\mathbf{x})}
$$

with two constant symmetric matrices $\mathbf{P}, \mathbf{T}$, and one positive semi-definite matrix $\mathbf{Q}$.

The following presents two models with different censoring plans. These two models will serve as two typical examples throughout this section and the sections hereafter in the context of all prediction, one-point and general extrapolation. Prior to introducing these models, we first describe the definitions of the two censoring plans involved in these upcoming models: (1) time (Type I) censoring is where the data are censored at a predefined time; (2) failure (Type II) censoring is where the data are censored after a prespecified number of failures. Detailed information about these and other types of censoring can be found in Nelson (1990).

Model 1: We suppose that the experimenter plans a design under the assumed regression model:

$$
E(Y \mid x)=\theta_{0}+\sum_{i=1}^{q} \theta_{i} x_{i}
$$

with $x_{i} \in\left[b_{i 1}, b_{i 2}\right]$, and employs time censoring. The data are collected at a fixed time $\zeta\left(x_{1}, x_{2}, \ldots, x_{q}\right) \equiv \zeta$ for all test units at all stress levels. Note that for life testing, $Y$ and $x_{i}^{\prime} s$ stand for the transformed lifetime and stresses, respectively. Such transformations are sometimes employed for the purpose of enhancing both linearity and normality simultaneously. So, it is sensible to consider that the regression model assumed above is approximately true.

Model 2: For the nonlinear regression model:

$$
E(Y \mid x) \approx h\left(\theta_{0}+\sum_{i=1}^{p-1} \theta_{i} x^{i}\right)
$$

where $h(z)=\mathrm{e}^{z}$, we suppose that the failure censoring is planned with a constant expected failure proportion of failures at all stress levels. We assume $\tau(x) \equiv \tau$. Namely, the experimenter expects about $100 \times \Phi(\tau) \%$ units fails at each stress level. So, $a(\tau)$ remains constant as well from its definition. We take $S=\left[b_{1}, b_{2}\right]$.

Example 1. According to Theorem 2, when $q=1$, the locally optimal robust prediction design for Model 1 is given by

$$
k(x)=\left[\frac{a_{1}+a_{2} x+a_{3} x^{2}}{a(\tau)\left(a_{4}+a_{5} x+a_{6} x^{2}\right)}+\frac{d}{a^{2}(\tau)\left(a_{4}+a_{5} x+a_{6} x^{2}\right)}\right]^{+},
$$

where $\tau=\left(\zeta-\theta_{0}-\theta_{1} x\right) / \sigma, a_{4}$ and $a_{6}$ are nonnegative and satisfy $4 a_{4} a_{6} \geqslant a_{5}^{2}$; when $q=2$,

$$
k\left(x_{1}, x_{2}\right)=\left[\frac{a_{1} x_{1}^{2}+a_{2} x_{1} x_{2}+a_{3} x_{2}^{2}}{a(\tau)\left(a_{4} x_{1}^{2}+a_{5} x_{1} x_{2}+a_{6} x_{2}^{2}\right)}+\frac{d}{a^{2}(\tau)\left(a_{4} x_{1}^{2}+a_{5} x_{1} x_{2}+a_{6} x_{2}^{2}\right)}\right]^{+},
$$

where $\tau=\left(\zeta-\theta_{0}-\theta_{1} x_{1}-\theta_{2} x_{2}\right) / \sigma, a_{4}$ and $a_{6}$ are nonnegative and satisfy $4 a_{4} a_{6} \geqslant a_{5}^{2}$. In addition, $a_{1}, a_{2}, a_{3}, a_{4}, a_{5}, a_{6}$ and $d$ are selected in order to minimize (5) subject to $\int_{b_{11}}^{b} k(x) \mathrm{d} x=1$ for the first case, $\int_{b_{21}}^{b_{22}} \int_{b_{11}}^{b_{12}} k\left(x_{1}, x_{2}\right) \mathrm{d} x_{1} \mathrm{~d} x_{2}=1$ for the second.

Example 2. For Model 2 with $p=2$, as a result of Theorem 2, the locally optimal design density for prediction is of the form

$$
k(x)=\left[\frac{a_{1}+a_{2} x+a_{3} x^{2}}{a_{4}+a_{5} x+a_{6} x^{2}}+\frac{d}{e^{2 \theta_{1} x}\left(a_{4}+a_{5} x+a_{6} x^{2}\right)}\right]^{+},
$$

where $a_{4} \geqslant 0, a_{6} \geqslant 0$, and $4 a_{4} a_{6} \geqslant a_{5}^{2}$. Besides, $a_{1}-a_{6}$ and $d$ are determined so as to minimize $(5)$ subject to $\int_{b_{1}}^{b_{2}} k(x) \mathrm{d} x=1$. The dependence of the design on $\theta_{1}$ makes such a design only locally optimal. This issue will be addressed in Section 5 .

\subsection{Minimax designs for one-point extrapolation: solutions to P2}

The loss function for Problem P2 becomes

$$
\begin{aligned}
I_{2} & =n E\left[h\left(\mathbf{z}^{\mathrm{T}}\left(\mathbf{x}_{0}\right) \hat{\boldsymbol{\theta}}\right)-h\left(\mathbf{z}^{\mathrm{T}}\left(\mathbf{x}_{0}\right) \boldsymbol{\theta}_{0}\right)-n^{-1 / 2} f\left(\mathbf{x}_{0}\right)\right]^{2} \\
& =\mathbf{b}_{f, S}^{\mathrm{T}} \mathbf{H}_{0}^{-1} \mathbf{b}_{f, S}-2 f\left(\mathbf{x}_{0}\right) \tilde{\mathbf{z}}^{\mathrm{T}}\left(\mathbf{x}_{0}\right) \mathbf{B}^{-1} \mathbf{b}_{f, S}+\sigma^{2} \tilde{\mathbf{z}}^{\mathrm{T}}\left(\mathbf{x}_{0}\right) \mathbf{B}^{-1} \tilde{\mathbf{z}}\left(\mathbf{x}_{0}\right)+f^{2}\left(\mathbf{x}_{0}\right) .
\end{aligned}
$$


Let $r_{\mathbf{x}_{0}, S}:=\eta_{0} / \eta_{S}$ represent the relative amount of model response uncertainty at the extrapolation point and within the design space. We define $\lambda_{k}^{(2)}=\tilde{\mathbf{z}}^{\mathrm{T}}\left(\mathbf{x}_{0}\right) \mathbf{B}^{-1} \mathbf{G B}^{-1} \tilde{\mathbf{z}}\left(\mathbf{x}_{0}\right)$, and $\mathbf{c}_{2}=\mathbf{B}^{-1} \tilde{\mathbf{z}}\left(\mathbf{x}_{0}\right) / \sqrt{\lambda_{k}^{(2)}}$. For a fixed $k(\mathbf{x})$, the maximization part of the minimax solutions to Problem P2 is given by Theorem 3.

Theorem 3. The maximum of $I_{2}$ is

$$
\sup _{f \in \mathscr{F}_{2}} I_{2}=\eta_{S}^{2}\left[\left(\sqrt{\lambda_{k}^{(2)}}+r_{0, S}\right)^{2}+v \tilde{\mathbf{z}}^{\mathrm{T}}\left(\mathbf{x}_{0}\right) \mathbf{B}^{-1} \tilde{\mathbf{z}}\left(\mathbf{x}_{0}\right)\right]
$$

attained at

$$
f_{k}(\mathbf{x})= \begin{cases}\eta_{S} \tilde{\mathbf{z}}^{\mathrm{T}}(\mathbf{x})\left[a(\mathbf{x}) k(\mathbf{x}) \mathbf{I}-\mathbf{A}_{S}^{-1} \mathbf{B}\right] \mathbf{c}_{2}, & \mathbf{x} \in S, \\ -\eta_{0}, & \mathbf{x}=\mathbf{x}_{0} .\end{cases}
$$

The minimax solution for P2 is presented in Theorem 4.

Theorem 4. The design density $k(\mathbf{x})$ minimizing (7) for one-point extrapolation is

$$
k(\mathbf{x})=\left[\frac{\mathbf{z}^{\mathrm{T}}(\mathbf{x}) \boldsymbol{\alpha}}{a(\mathbf{x}) \mathbf{z}^{\mathrm{T}}(\mathbf{x}) \boldsymbol{\beta}}+\frac{\lambda}{a^{2}(\mathbf{x})\left[\tilde{\mathbf{z}}^{\mathrm{T}}(\mathbf{x}) \boldsymbol{\beta}\right]^{2}}\right]^{+}
$$

for some $p \times 1$ vectors $\boldsymbol{\alpha}, \boldsymbol{\beta}$ and constant $\lambda$ which satisfy: (i) $\int_{S} k(\mathbf{x}) \mathrm{d} \mathbf{x}=1$, (ii) minimize (7).

Example 3. Recall Model 1 with $q=1$. Suppose that the estimation extrapolates to one point $x_{0}>b_{12}$ or $x_{0}<b_{11}$. According to Theorem 4, the locally optimal robust one-point extrapolation design for this model is given by

$$
k(x)=\left[\frac{a_{1} x+a_{2}}{a(\tau)\left(a_{3} x+a_{4}\right)}+\frac{a_{5}}{\left\{a(\tau)\left(a_{3} x+a_{4}\right)\right\}^{2}}\right]^{+},
$$

where $\tau=\left(\zeta-\theta_{0}-\theta_{1} x\right) / \sigma$, and $a_{1}-a_{5}$ are chosen in order to minimize (7) subject to $\int_{b_{11}}^{b_{12}} k(x) \mathrm{d} x=1$.

When $q=2$, suppose that the estimation extrapolates to one point $\mathbf{x}_{0}=\left(x_{10}, x_{20}\right)$ with $x_{10}>b_{12}$ and $x_{20}>b_{22}$; or $x_{10}<b_{11}$ and $x_{20}<b_{21}$. According to Theorem 4, the locally optimal robust one-point extrapolation design for this model is given by

$$
k\left(x_{1}, x_{2}\right)=\left[\frac{a_{1} x_{1}+a_{2} x_{2}+a_{3}}{a(\tau)\left(a_{4} x_{1}+a_{5} x_{2}+a_{6}\right)}+\frac{a_{7}}{\left\{a(\tau)\left(a_{4} x_{1}+a_{5} x_{2}+a_{6}\right)\right\}^{2}}\right]^{+},
$$

where $\tau=\left(\zeta-\theta_{0}-\theta_{1} x_{1}-\theta_{2} x_{2}\right) / \sigma$, and $a_{1}-a_{7}$ are chosen in order to minimize (7) subject to $\int_{b_{21}}^{b_{22}} \int_{b_{11}}^{b_{12}} k\left(x_{1}, x_{2}\right) \mathrm{d} x_{1} \mathrm{~d} x_{2}=1$.

Example 4. We revisit Model 2 with $x_{0}>b_{2}$ or $x_{0}<b_{1}$. When $p=2$, the locally optimal design density for one-point extrapolation is given by

$$
k(x)=\left[\frac{a_{1} x+a_{2}}{a_{3} x+a_{4}}+\frac{a_{5}}{e^{2 \theta_{1} x}\left(a_{3} x+a_{4}\right)^{2}}\right]^{+},
$$

where $a_{1}-a_{5}$ are selected by minimizing (7) subject to $\int_{b_{1}}^{b_{2}} k(x) \mathrm{d} x=1$. The computation of the numerical values for $a_{1}-a_{5}$ in this design will be presented in Section 5 .

When $p=3$, the locally optimal design density for one-point extrapolation is given by

$$
k(x)=\left[\frac{a_{1}+a_{2} x+a_{3} x^{2}}{a_{4}+a_{5} x+a_{6} x^{2}}+\frac{a_{7}}{e^{2 \theta_{1} x}\left(a_{4}+a_{5} x+a_{6} x^{2}\right)^{2}}\right]^{+},
$$

where $a_{1}-a_{7}$ are selected by minimizing (7) subject to $\int_{b_{1}}^{b_{2}} k(x) \mathrm{d} x=1$. 
3.3. Minimax designs for general extrapolation: solutions to P3

The loss function for Problem P3 is

$$
\begin{aligned}
I_{2} & =n \int_{T} E\left[h\left(\mathbf{z}^{\mathrm{T}}(\mathbf{x}) \hat{\boldsymbol{\theta}}\right)-h\left(\mathbf{z}^{\mathrm{T}}(\mathbf{x}) \boldsymbol{\theta}_{0}\right)-n^{-1 / 2} f(\mathbf{x})\right]^{2} \mathrm{~d} \mathbf{x} \\
& =\mathbf{b}_{f, S}^{\mathrm{T}} \mathbf{H}_{T} \mathbf{b}_{f, S}-2 \mathbf{b}_{f, T}^{\mathrm{T}} \mathbf{B}^{-1} \mathbf{b}_{f, S}+\sigma^{2} \operatorname{tr}\left(\mathbf{A}_{T} \mathbf{B}^{-1}\right)+\int_{T} f^{2}(\mathbf{x}) \mathrm{d} \mathbf{x} .
\end{aligned}
$$

We denote $r_{T, S}:=\eta_{T} / \eta_{S}$ for the relative amount of model response uncertainty in the extrapolation and design spaces. We also denote $\lambda_{k}^{(3)}$ to be the largest solution to $\left|\mathbf{G}-\lambda \mathbf{H}_{T}\right|=0$ and let $\mathbf{c}_{3}$ be any vector satisfying $\left(\mathbf{G} \mathbf{H}_{T} \mathbf{G}-\lambda_{k}^{(3)} \mathbf{G}\right) \mathbf{c}=0$ and (4). The maximum of $I_{2}$ is given by Theorem 5 .

Theorem 5. The maximum of $I_{2}$ is

$$
\sup _{f \in \mathscr{F}_{3}} I_{2}(f, \xi)=\eta_{S}^{2}\left[\left(\sqrt{\lambda_{k}^{(3)}}+r_{T, S}\right)^{2}+v \operatorname{tr}\left(\mathbf{A}_{T} \mathbf{B}^{-1}\right)\right]
$$

attained at

$$
f_{k}(\mathbf{x})= \begin{cases}\eta_{S} \tilde{\mathbf{z}}^{\mathrm{T}}(\mathbf{x})\left[a(\mathbf{x}) k(\mathbf{x}) \mathbf{I}-\mathbf{A}_{S}^{-1} \mathbf{B}\right] \mathbf{c}_{3}, & \mathbf{x} \in S, \\ -\frac{\eta_{T} \tilde{\mathbf{z}}^{\mathrm{T}}(\mathbf{x}) \mathbf{B}^{-1} \mathbf{G} \mathbf{c}_{3}}{\sqrt{\lambda_{k}^{(3)}}}, & \mathbf{x} \in T .\end{cases}
$$

The following theorem gives the optimal minimax design density for the general extrapolation Problem P3, which has the same form as (6) for P1.

Theorem 6. The design density $k(\mathbf{x})$ minimizing (10) for general extrapolation is

$$
k(\mathbf{x})=\frac{\left[a(\mathbf{x}) \tilde{\mathbf{z}}^{\mathrm{T}}(\mathbf{x}) \mathbf{P} \tilde{\mathbf{z}}(\mathbf{x})+d\right]^{+}}{a^{2}(\mathbf{x}) \tilde{\mathbf{z}}^{\mathrm{T}}(\mathbf{x}) \mathbf{Q} \tilde{\mathbf{z}}(\mathbf{x})}
$$

for some constant symmetric matrix $\mathbf{P}$, a positive semi-definite matrix $\mathbf{Q}$, and a constant $d$ that minimize $(10)$ and satisfy $\int_{S} k(\mathbf{x}) \mathrm{d} \mathbf{x}=1$.

Example 5. For Model 1 with $q=1$, and in the context of general extrapolation with extrapolation space $T=\left[t_{1}, t_{2}\right] \backslash\left[b_{11}, b_{12}\right]$, as a result of Theorem 6 , the locally optimal robust extrapolation design for such model is of the form

$$
k(x)=\left[\frac{\left(a_{1}+a_{2} x+a_{3} x^{2}\right)}{\left(a_{4}+a_{5} x+a_{6} x^{2}\right)}+\frac{d}{a(\tau)\left(a_{4}+a_{5} x+a_{6} x^{2}\right)}\right]^{+},
$$

where $a_{4}$ and $a_{6}$ are nonnegative and satisfy $4 a_{4} a_{6} \geqslant a_{5}^{2}$. Moreover, $a_{1}-a_{6}$ and $d$ are chosen to minimize (10) subject to $\int_{b_{11}}^{b_{12}} k(x) \mathrm{d} x=1$

Example 6. With Model 2, when $p=2$, in the context of general extrapolation with extrapolation space $T=\left[t_{1}, t_{2}\right] \backslash\left[b_{1}, b_{2}\right]$, by Theorem 6 the locally optimal design density for general extrapolation is given by

$$
k(x)=\left[\frac{e^{2 \theta_{1} x}\left(a_{1}+a_{2} x+a_{3} x^{2}\right)+d}{e^{2 \theta_{1} x}\left(a_{4}+a_{5} x+a_{6} x^{2}\right)}\right]^{+},
$$

where $a_{4} \geqslant 0, a_{6} \geqslant 0,4 a_{4} a_{6} \geqslant a_{5}^{2}$, and $a_{1}-a_{6}$, as well as $d$ are selected so as to minimize (10) conditional on $\int_{b_{1}}^{b_{2}} k(x) \mathrm{d} x=1$.

\section{Unbiased designs for censored data: solutions to P4-P6}

For each case of P4-P6, we denote the bias component caused by model misspecification within the design space:

$$
I B_{1}(f, \xi, \zeta)=\mathbf{b}_{f, S}^{\mathrm{T}} \mathbf{H}_{S}^{-1} \mathbf{b}_{f, S}
$$

for P4,

$$
I B_{2}(f, \xi, \zeta)=\mathbf{b}_{f, S}^{\mathrm{T}} \mathbf{H}_{0}^{-1} \mathbf{b}_{f, S}-2 f\left(\mathbf{x}_{0}\right) \tilde{\mathbf{z}}^{\mathrm{T}}\left(\mathbf{x}_{0}\right) \mathbf{B}^{-1} \mathbf{b}_{f, S}
$$


for P5, and

$$
I B_{3}(f, \xi, \zeta)=\mathbf{b}_{f, S}^{\mathrm{T}} \mathbf{H}_{T} \mathbf{b}_{f, S}-2 \mathbf{b}_{f, T}^{\mathrm{T}} \mathbf{B}^{-1} \mathbf{b}_{f, S}
$$

for P6. We say that a design/censoring pair $(\xi, \zeta)$ is unbiased if it satisfies one of

$$
I B_{i}(f, \xi, \zeta)=0 \quad \text { for all } f \in \mathscr{F}_{i}, \quad i=1,2 \text {, or } 3 \text {. }
$$

Therefore, we have $\sup _{f} I B_{i}(f, \xi, \zeta)=0$ for $i=1,2$, or 3 .

Therefore, $\mathrm{P} 4, \mathrm{P} 5$, or $\mathrm{P} 6$ has become to find the design such that the maximum, over $f$, of its matching bias: $\sup _{f} I B_{i}(f, \xi, \zeta)$ for $i=1,2$, or 3 is zero. Let $\alpha=\left\{\int_{S} a^{-1}(\mathbf{x}) \mathrm{d} \mathbf{x}\right\}^{-1}$. The following theorem offers a necessary and sufficient condition for unbiasedness, the unbiased designs and the resulting losses, respectively.

Theorem 7. (a) The design $k(\mathbf{x})$ is unbiased if and only if

$$
a(\mathbf{x}) k(\mathbf{x}) \equiv \alpha
$$

(b) The unbiased design density is

$$
k(\mathbf{x})=\alpha a^{-1}(\mathbf{x})
$$

(c) The corresponding losses under unbiased designs are as follows:

(i) for prediction,

$$
I_{1}=\eta_{S}^{2}+\alpha p \sigma^{2}
$$

(ii) for one-point extrapolation at $\mathbf{x}_{0}$,

$$
I_{2}=\eta_{T}^{2}+\alpha \sigma^{2} \tilde{\mathbf{z}}^{\mathrm{T}}\left(\mathbf{x}_{0}\right) \mathbf{A}_{S}^{-1} \tilde{\mathbf{z}}\left(\mathbf{x}_{0}\right)
$$

(iii) for general extrapolation,

$$
I_{2}=\eta_{T}^{2}+\alpha \sigma^{2} \operatorname{tr}\left(\mathbf{A}_{T} \mathbf{A}_{S}^{-1}\right) .
$$

We notice that the unbiased designs for the cases of prediction, one-point and general extrapolations are the same.

Example 7. With Model 1 and $q=1$, as stated in Theorem 6, the locally unbiased design is of the form

$$
k(x)=a^{-1}(x)\left\{\int_{b_{11}}^{b_{12}} a^{-1}(x) \mathrm{d} x\right\}^{-1}
$$

where

$$
a(x)=\Phi\left(\frac{\zeta-\theta_{0}-\theta_{1} x}{\sigma}\right)-\frac{\zeta-\theta_{0}-\theta_{1} x}{\sigma} \phi\left(\frac{\zeta-\theta_{0}-\theta_{1} x}{\sigma}\right)+\frac{\phi^{2}\left(\left(\zeta-\theta_{0}-\theta_{1} x\right) / \sigma\right)}{1-\Phi\left(\left(\zeta-\theta_{0}-\theta_{1} x\right) / \sigma\right)} .
$$

When $q=2$, the locally unbiased design is of the form

$$
k\left(x_{1}, x_{2}\right)=a^{-1}\left(x_{1}, x_{2}\right)\left\{\int_{b_{21}}^{b_{22}} \int_{b_{11}}^{b_{12}} a^{-1}\left(x_{1}, x_{2}\right) \mathrm{d} x_{1} \mathrm{~d} x_{2}\right\}^{-1}
$$

where

$$
a\left(x_{1}, x_{2}\right)=\Phi\left(\frac{\zeta-\theta_{0}-\theta_{1} x_{1}-\theta_{2} x_{2}}{\sigma}\right)-\frac{\zeta-\theta_{0}-\theta_{1} x_{1}-\theta_{2} x_{2}}{\sigma} \phi\left(\frac{\zeta-\theta_{0}-\theta_{1} x_{1}-\theta_{2} x_{2}}{\sigma}\right)+\frac{\phi^{2}\left(\left(\zeta-\theta_{0}-\theta_{1} x_{1}-\theta_{2} x_{2}\right) / \sigma\right)}{1-\Phi\left(\left(\zeta-\theta_{0}-\theta_{1} x_{1}-\theta_{2} x_{2}\right) / \sigma\right)} .
$$

Example 8. For Model 2, the unbiased robust design is uniform with density $k(x)=\left\{\int_{S} \mathrm{~d} x\right\}^{-1}$ since $a(x)$ is constant in this model. 


\section{Computation}

In this section, we demonstrate the computation of numerical values of the constants in our constructed designs using one typical ALT example: Model 2 with one-point extrapolation when $p=2$.

As indicated in Example 4, the locally optimal design density for one-point extrapolation is given by $(8)$. We assume $S=[0,1]$ and $x_{0}>1$. We also let $\tau$ be a predefined constant which is essentially the standard normality quantile corresponding to the expected percentage of failures. For instance, if the experimenter plans to employ Type II censoring and expects 70\% of the units to fail at each stress level, $\tau=\Phi^{-1}(0.7)=0.525$. Then, $a(\tau)$ remains constant given by $a(0.525)=0.921$.

For Model 2, since the constant term $a(\tau)$ has been cancelled out in the first term of (7), it is only involved in the second term of (7). Let $\rho=v / a(\tau)$. For prespecified model parameters, the design varies when $x_{0}, r_{x_{0}, S}$ or $\rho$ changes. All of $x_{0}, r_{\mathbf{x}_{0}, S}$ and $\rho$ can be determined by the experimenter. We also notice that the term $e^{2 \theta_{0}}$ has been cancelled out in both the first and second terms of (7). However, for fixed $x_{0}, r_{x_{0}, S}$ and $\rho$, the optimal design depends still on the value of $\theta_{1}$, therefore, is only locally optimal. To deal with this issue, we search for "locally most robust" designs as discussed in Wiens and Xu (2008a). To do this, firstly we obtain the local optimal design for an initial value of $\theta_{1}$; secondly, for this optimal design, we take a further maximum of the loss as $\theta_{1}$ varies over some interval $I$, and record the least favorable $\theta_{1}$, written $\theta_{1}^{\mathrm{LF}}$; thirdly, we determine the coefficients of $k(x)$ so as to minimize this maximum loss for $\theta_{1}^{\mathrm{LF}}$. We repeat this procedure until $\theta_{1}^{\mathrm{LF}}$ converges. A stopping rule for the convergences is applied. For the minimization part, the minimizer $\left(a_{1}, a_{2}, a_{3}, a_{5}\right)$ converges when one of the following two situations occurs: the loss reduction being no more than $t^{2 / 3}$ or maximum of the absolute changes in all the arguments $a_{1}, a_{2}, a_{3}, a_{5}$ being smaller than $\sqrt{t}$, where $t$ is a machine epsilon; for the maximization, the maximizer $\theta_{1}$ converges when one of the following situations occurs: the loss increment being no more than $t^{2 / 3}$ or the absolute change in $\theta_{1}$ being smaller than $\sqrt{t}$, where $t$ is a machine epsilon. In the example below, we take $t=2^{-23}$.

To illustrate the approach described above, we consider the Class-B insulation data from Nelson (1990, Table 4.1 of Chapter 3 ). Those data are collected from a singly time-censored ALT conducted using a uniform design on four specified testing levels. The acceleration stress is temperature. The intention of this experiment is to estimate the lifetime for electric motors at the normal usage temperature of $130^{\circ} \mathrm{C}$. The failures obtained from this test are at stress levels ranging from 170 to $220^{\circ} \mathrm{C}$. The Arrhenius-lognormal model is fitted for those data and the MLEs for the model parameters are located by Nelson (1990). The transformation of the lifetime used in this example is the logarithm and that of stress $t$ is 1000 times the inverse of the absolute temperature in degrees Kelvin, i.e. $x^{\prime}=1000 /(t+273.16)$. For simplicity, we transform $x^{\prime}$ to our stress variable $x$ with domain of $[0,1]$ through the linear transformation $x=\left(-2.028+x^{\prime}\right) / 0.229$. Under such transformations, the MLE of $\theta_{1}$ for the nominal

Table 1

Numerical values for (8)

\begin{tabular}{llll}
\hline$\rho$ & $a_{1}$ & $a_{2}$ & $a_{3}$ \\
\hline$S=[0,1], \theta_{1}=0.987, r_{x_{0}, S}=1$, and $x_{0}=1.98$. & & \\
0.5 & -0.190 & 0.0003 & -0.730 \\
1 & -0.420 & 0.002 & -0.806 \\
2 & -0.676 & 0.00003 & -0.858 \\
\hline
\end{tabular}

a

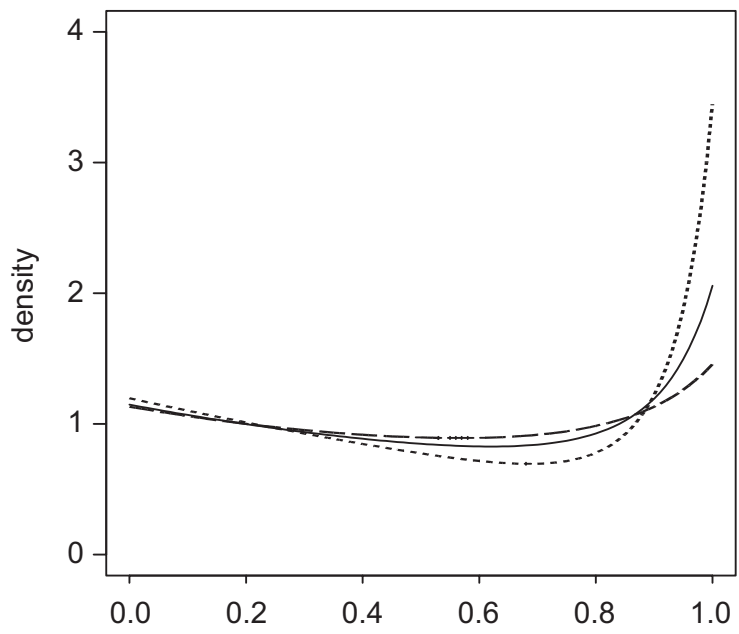

b

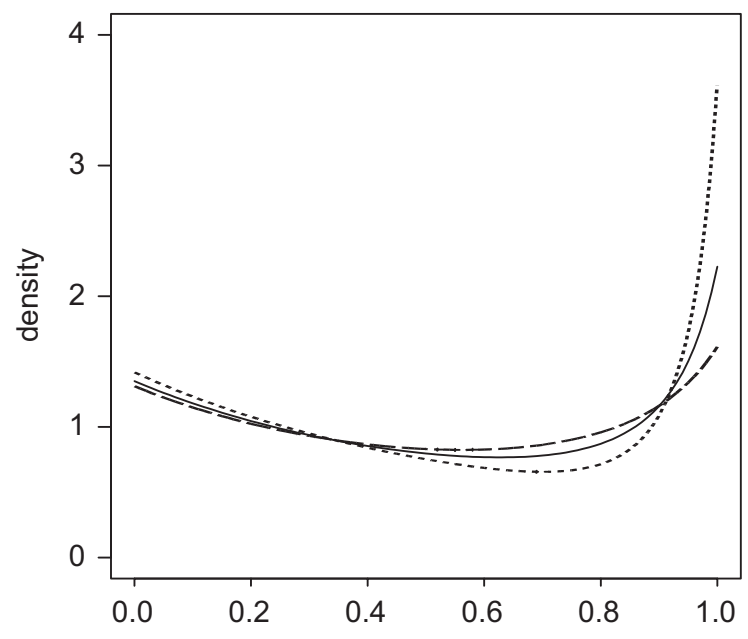

Fig. 1. Optimal design densities $k(x)=\left[\frac{a_{1} x+a_{2}}{a_{3} x+a_{4}}+\frac{a_{5}}{e^{2 \theta_{1} x}\left(a_{3} x+a_{4}\right)^{2}}\right]^{+}$for $S=[0,1], r_{x_{0}, S}=1$, and $x_{0}=1.98$. (a) locally optimal design densities for $\theta_{1}=0.987$; (b) locally most robust design densities for $\theta_{1}$ within $I=[0.5,1.5]$. Each plots uses three values of $\rho$ : $\rho=1$ (solid line), $\rho=0.5$ (broken line), $\rho=2$ (dotted line). 
Table 2

Numerical values for (8)

\begin{tabular}{lllll}
\hline$\rho$ & $a_{1}$ & $a_{2}$ & $a_{3}$ & $\theta_{1}^{\mathrm{LF}}$ \\
\hline$S=[0,1], \theta_{1} \in[0.5,1.5], r_{x_{0}, S=1, \text { and } x_{0}=1.98}$ & & & \\
0.5 & 0.049 & 0.001 & -0.783 & 1.311 \\
1 & -0.142 & 0.002 & -0.855 & 1.349 \\
2 & -0.318 & 0.00003 & -0.897 & 1.5 \\
\hline
\end{tabular}

Table 3

Relative efficiencies for locally optimal designs and locally most robust designs with $S=[0,1], r_{x_{0}, S}=1$, and $x_{0}=1.98$

\begin{tabular}{|c|c|c|c|c|c|c|c|c|}
\hline \multirow[t]{3}{*}{$\rho$} & \multicolumn{4}{|c|}{ True $\theta_{1}$ being 0.987} & \multicolumn{4}{|c|}{ True $\theta_{1}$ being 1.5} \\
\hline & \multicolumn{2}{|c|}{ Case (1) } & \multicolumn{2}{|c|}{ Case (2) } & \multicolumn{2}{|c|}{ Case (3) } & \multicolumn{2}{|c|}{ Case (4) } \\
\hline & Local & Most & Local & Most & Local & Most & Local & Most \\
\hline 0.5 & 1 & 0.990 & 0.334 & 0.838 & 0.991 & 1 & 0.338 & 0.841 \\
\hline 1 & 1 & 0.993 & 0.055 & 0.679 & 0.994 & 1 & 0.044 & 0.673 \\
\hline 2 & 1 & 0.974 & 0.029 & 0.586 & 0.979 & 1 & 0.020 & 0.556 \\
\hline
\end{tabular}

model is $\hat{\theta}_{1}=0.987$ and the corresponding $99 \%$ confidence interval for $\theta_{1}$ is $(0.730,1.243)$. Taking the model misspecification into account, we consider an even broader region $\theta_{1} \in I=[0.5,1.5]$. We apply the same extrapolation point $x_{0}=1.98$ as employed in Nelson (1990), which is equivalent to the normal usage temperature: $t_{0}=130^{\circ} \mathrm{C}$.

It should be noted that in (8), if one of $a_{1}-a_{5}$ is nonzero, then we can assume that it is 1 . In the following computation we take $a_{4}=1$. For locally optimal designs when $\theta_{1}=0.987$, see Table 1 for the numerical values of the constants in (8) with various $\rho$ and Fig. 1(a) for the plots. For locally most robust designs, we carry out the process described above for $I=[0.5,1.5]$ and several $\rho$, each time starting at $\theta_{1}=0.987$. The locally most robust designs are detailed in Table 2 . For these locally optimal designs, the corresponding maximum loss functions are nondecreasing functions with respect to $\theta_{1}$. In each case, we find that the least favorable $\theta_{1}$ are attained at the upper bound of $I$ : 1.5 . See Fig. 1(b) for the plots. All plots use $a_{4}=1$ and $r_{x_{0}, S}=1$. The Splus code for finding the optimal robust design is available at author's website: http://spartan.ac.brocku.ca/ Xxu/find_robust_designs.SSC.

In order to compare the relative efficiencies of these two classes of designs, we consider the following four cases: (1) both true and assumed $\theta_{1}$ being 0.987 , (2) true $\theta_{1}$ being 0.987 but assumed $\theta_{1}$ being $1.5,(3)$ both true and assumed $\theta_{1}$ being 1.5 , ( 4 ) true $\theta_{1}$ being 1.5 but assumed $\theta_{1}$ being 0.987 . We define that the relative efficiency is the ratio of the loss for the case of the assumed $\theta_{1}$ being correct and the loss for that the corresponding case of the assumed $\theta_{1}$ being wrong. Table 3 provides the values of the relative efficiencies for both types of designs shown in Tables 1 and 2. The densities for locally optimal designs and locally most robust designs are considerably similar in Fig. 1 . Consequently, when the specifications in $\theta_{1}$ are correct, the efficiency of the two designs are fairly close (see Cases (1) and (3) in Table 3). However, for all other cases the relative efficiencies for locally optimal designs are below 35\%. It shows that the locally optimal designs (denoted by "Local") lose their efficiencies rapidly once $\theta_{1}$ is misspecified. On the other hand, the relative efficiencies of the most robust designs (Most) remains above 55\% for all cases with a misspecified $\theta_{1}$. This indicates that the most robust deigns should be considered when the experimenter suspects possible moderate misspecification.

We note that for the extreme case, when the data are complete, $\tau \longrightarrow \infty$, we have $a(\tau)=1$. In this case, the results obtained in Section 3 for this extreme case degenerate into the exact optimal robust designs for complete data which are presented in Wiens and Xu (2008a, b).

\section{Implementation}

In this section, we discuss some implementation schemes that are utilized to approximate a robust design having a density, which in practice is not implementable. We also confirm that one of the matching quantile schemes used in the literature is optimal with respect to certain criteria. In addition, using this optimal implementation schemes proposed, the resulting implementable designs for the designs which have obtained in the previous section are given.

In the preceding section of the present paper, we have obtained a number of robust designs for various cases which turned out to have densities. Such designs with densities are prevalent in the literature. See, for instance, Huber (1975), Wiens (1990, 1992), Wiens and Xu (2008a, b). In addition, Heo (1998) listed quite a few such continuous designs. Wiens (1992) showed that if the contamination class is an $L_{2}$-type of space, e.g. Class $\mathscr{F}_{i}(i=1,2,3)$ in Section 2 , any optimal design minimizing the maximum loss function over such contamination space has to be absolutely continuous. In practice, a design should tell the experimenter what the design support points are and how many subjects should be allocated to each of these points. An implementable design, $\xi$, must be a discrete probability measure which puts probability $\xi\left(\mathbf{x}_{i}\right)$ at $\mathbf{x}_{i}$. For a given $r \leqslant n$, let $\mathbf{x}_{1}, \mathbf{x}_{2}, \ldots, \mathbf{x}_{r}$ be the distinct design points, the number of subjects allocated to a particular design point $\mathbf{x}_{i}$ on design space is then, $n \xi\left(\mathbf{x}_{i}\right)$. In addition, each $\xi\left(\mathbf{x}_{i}\right), i=1,2, \ldots, r$, should be an integer multiple of $1 / n$. A design with this integer property is called an exact design. 
Table 4

Exact design temperatures $t\left({ }^{\circ} \mathrm{C}\right)$ which approximate the designs presented in Table 1

\begin{tabular}{|c|c|c|c|c|c|c|c|c|c|c|}
\hline \multirow{2}{*}{$\begin{array}{l}\rho \rho \\
0.5\end{array}$} & \multicolumn{10}{|c|}{ Design points for $n=10, t \in\left[170^{\circ} \mathrm{C}, 220^{\circ} \mathrm{C}\right], \theta_{1}=0.987, r_{x_{0}, s}=1$, and $t_{0}=130^{\circ} \mathrm{C}$} \\
\hline & 171.53 & 175.39 & 179.95 & 185.05 & 190.48 & 196.04 & 201.59 & 207.04 & 212.33 & 217.45 \\
\hline 1 & 171.19 & 174.4 & 178.8 & 184.18 & 190 & 195.87 & 201.60 & 207.12 & 212.42 & 217.48 \\
\hline 2 & 170.68 & 172.82 & 176.58 & 182.57 & 189.42 & 195.92 & 201.91 & 207.47 & 212.67 & 217.58 \\
\hline
\end{tabular}

Table 5

Exact design temperatures $t\left({ }^{\circ} \mathrm{C}\right)$ which approximate the designs presented in Table 2

\begin{tabular}{|c|c|c|c|c|c|c|c|c|c|c|}
\hline \multirow{2}{*}{$\frac{\rho}{0.5}$} & \multicolumn{10}{|c|}{ Design points for $n=10, t \in\left[170^{\circ} \mathrm{C}, 220^{\circ} \mathrm{C}\right], \theta_{1} \in[0.5,1.5], r_{x_{0}, S}=1, t_{0}=130^{\circ} \mathrm{C}$} \\
\hline & 171.43 & 175.07 & 179.60 & 184.93 & 190.77 & 196.76 & 202.58 & 208.06 & 213.12 & 217.77 \\
\hline 1 & 171.06 & 174.22 & 178.83 & 184.64 & 190.95 & 197.17 & 203.02 & 208.40 & 213.33 & 217.83 \\
\hline 2 & 170.58 & 172.87 & 177.36 & 184.20 & 191.36 & 197.88 & 203.7 & 208.92 & 213.64 & 217.93 \\
\hline
\end{tabular}

Since a design with density is not an exact design, a few implementation approaches have been introduced in the literature. Wiens (1992) suggested a randomized design. That is, the design points are randomly chosen from an optimal design density. Wiens and Zhou (1996) presented a more systematic approach of matching quantiles that places an equal number of observations at the quantiles of an optimal design density. This type of approach has been used in both Heo (1998) and Fang (1999). Matching moments is another method that chooses design points such that the empirical moments match up as closely as possible with the theoretical moments, obtained from the optimal density, to a sufficiently high order. This approach has been seen in Heo et al. (2001) and Adewale (2002). An innovation for obtaining an exact design was presented by Fang and Wiens (2000), who redefined the problem by considering a finite design space and applying the simulated annealing algorithm. See also most recently in Adewale and Wiens (2006).

The following discusses an optimal approximation for one-dimensional design space. There are two kinds of matching quantile approaches appearing in the literature. In the first, used in Wiens and Zhou (1996), one observation is placed at each of the following quantiles:

$$
x_{i}^{(1)}=F_{\xi_{0}}^{-1}\left(\frac{i-0.5}{n}\right), \quad i=1,2, \ldots, n,
$$

where $\xi_{0}$ is an optimal design with a density, and $F_{\xi_{0}}$ is the cumulative distribution function of $\xi_{0}$. In the second, used in Heo et al. (2001), one observation is placed at

$$
x_{i}^{(2)}=F_{\xi_{0}}^{-1}\left(\frac{i-1}{n-1}\right), \quad i=1,2, \ldots, n
$$

The first approach provides a sample from $\xi_{0}$ with a smaller Kolmogorov-Smirnov statistic, since

$$
\max _{1 \leqslant i \leqslant n}\left|F_{\xi_{0}}\left(x_{i}^{(1)}\right)-\frac{t_{i}}{n}\right|=\frac{1}{2 n}
$$

and

$$
\max _{1 \leqslant i \leqslant n}\left|F_{\xi_{0}}\left(x_{i}^{(2)}\right)-\frac{t_{i}}{n}\right|=\frac{1}{n},
$$

where $t_{i}$ is the number of design points less than $x_{i}$. For a fixed number of experimental subjects $n$, the following theorem shows that (11) is optimal under the defined criteria below.

Theorem 8. The first matching quantile approach provided by (11) offers an optimal approximation which minimizes the overall "distance" between the optimal design $\xi_{0}$ and the resulting one when this distance is defined as

$$
D=\int_{S}\left|F_{\xi_{0}}(x)-F_{\xi}(x)\right|^{m} \mathrm{~d} x
$$

for any positive $m$, among all the designs of the form of $\xi(x)=(1 / n) \sum_{i=1}^{n} I\left(x=x_{i}\right)$.

Let us take $n=10$ as a simple implementation exercise. We use (11) to obtain the exact designs in term of points $x^{\prime} s$ within design space $S=[0,1]$. Then, we transform these points into the design temperatures (stress levels) in term of $t$ ( ${ }^{\circ} \mathrm{C}$ ). See Tables 4 and 5 for the results. 


\section{Acknowledgments}

This study is supported by the Natural Sciences and Engineering Research Council of Canada (NSERC). The author is grateful to Douglas Wiens for his helpful suggestions, and to two anonymous reviewers for their insightful comments.

\section{Appendix A. List of symbols}

$Y$ response variable

$\mathbf{x}$ q-dimensional explanatory variable vector

$\mathbf{x}_{0}$ extrapolation point

$\sigma^{2}$ variance of $Y$

$f \sqrt{n}\left[E(Y \mid \mathbf{x})-h\left(\mathbf{z}^{\mathrm{T}}(\mathbf{x}) \boldsymbol{\theta}_{0}\right)\right.$ : contaminant

$\mu \mathbf{z}^{\mathrm{T}}(\mathbf{x}) \boldsymbol{\theta}$

$I_{1} n \int_{S} E\left[h\left(\mathbf{z}^{\mathrm{T}}(\mathbf{x}) \hat{\boldsymbol{\theta}}\right)-E(Y \mid \mathbf{x})^{2} \mathrm{~d} \mathbf{x}\right.$ prediction loss

$I_{3} n \int_{T} E\left[h\left(\mathbf{z}^{\mathrm{T}}(\mathbf{x}) \hat{\boldsymbol{\theta}}\right)-E(Y \mid \mathbf{x})^{2} \omega(d \mathbf{x})\right.$ : general extrapolation loss

$c$ an indicator function for censoring, defined by $c(y \mid \mathbf{x})= \begin{cases}1 & \text { when } y(\mathbf{x}) \leqslant \zeta(\mathbf{x}), \\ 0 & \text { when } y(\mathbf{x})>\zeta(\mathbf{x}) .\end{cases}$

$\phi$ standard normal density

$\Phi$ standard normal cumulative distribution function

$l_{i} \log$ likelihood of the $i$ th individual observation

$\mathbf{A}_{S} \int_{S} \tilde{\mathbf{z}}(\mathbf{x}) \tilde{\mathbf{z}}^{\mathrm{T}}(\mathbf{x}) \mathrm{d} \mathbf{x}$

$\mathbf{A}_{T} \int_{T} \tilde{\mathbf{z}}(\mathbf{x}) \tilde{\mathbf{z}}^{\mathrm{T}}(\mathbf{x}) \mathrm{d} \mathbf{x}$

$\mathbf{H}_{S} \mathbf{B}^{-1} \mathbf{A}_{S} \mathbf{B}^{-1}$

$\mathbf{H}_{T} \mathbf{B}^{-1} \mathbf{A}_{T} \mathbf{B}^{-1}$

$\mathbf{b}_{f, T} \int_{T} \tilde{\mathbf{z}}(\mathbf{x}) f(\mathbf{x}) \mathrm{d} \mathbf{x}$

$\tilde{\mathbf{b}}_{f}\left(\boldsymbol{\theta}_{0}\right)$ asymptotic expectation of the score function evaluated at $\boldsymbol{\theta}_{0}$

$\hat{\theta}$ maximum likelihood estimate of $\theta_{0}$

$\zeta$ censoring time

$S$ design space

$T$ extrapolation region

$\boldsymbol{\theta}_{0} \arg \min _{t} \int_{S}\left[E(Y \mid \mathbf{x})-h\left(\mathbf{z}^{\mathrm{T}}(\mathbf{x}) \mathbf{t}\right)^{2} \mathrm{~d} \mathbf{x}\right.$ : true value of regression parameters

$\mathbf{z}(\mathbf{x})$ p-dimentional regressor vector

$\tilde{\mathbf{z}}(\mathbf{x})\left(\left.\frac{\mathrm{d} h}{\mathrm{~d} \mu}\right|_{\boldsymbol{\theta}=\boldsymbol{\theta}_{0}}\right) \mathbf{z}(\mathbf{x})$

$I_{2} n E\left[h\left(\mathbf{z}^{\mathrm{T}}\left(\mathbf{x}_{0}\right) \hat{\boldsymbol{\theta}}\right)-E\left(Y \mid \mathbf{x}_{0}\right)^{2}\right.$ : one-point extrapolation loss

$\xi \sum_{i=1}^{n} I\left(\mathbf{x}=\mathbf{x}_{i}\right)$ : design measure, where $I$ is the indicator function

$w_{i} \frac{y\left(\mathbf{x}_{i}\right)-h\left(\mathbf{z}^{\mathrm{T}}\left(\mathbf{x}_{i}\right) \boldsymbol{\theta}_{0}\right)}{\sigma}$ : standardized observation at specified stress level $\mathbf{x}_{i}$

$\tau_{i} \frac{\zeta\left(\mathbf{x}_{i}\right)-h\left(\mathbf{z}^{\mathrm{T}}\left(\mathbf{x}_{i}\right) \boldsymbol{\theta}_{0}\right)}{\sigma}:$ standardized censoring time at specified stress level $\mathbf{x}_{i}$

$\eta_{S}$ upper bound of $|f|$ within $S$

$\mathbf{A}_{0} \tilde{\mathbf{z}}\left(\mathbf{x}_{0}\right) \tilde{\mathbf{z}}^{\mathrm{T}}\left(\mathbf{x}_{0}\right)$

B $\int_{S} a(\mathbf{x}) \tilde{\mathbf{z}}(\mathbf{x}) \tilde{\mathbf{z}}^{\mathrm{T}}(\mathbf{x}) \xi(\mathrm{d} \mathbf{x})$

$\mathbf{H}_{0} \mathbf{B}^{-1} \mathbf{A}_{0} \mathbf{B}^{-1}$

$\mathbf{b}_{f, S} \int_{S} a(\mathbf{x}) \tilde{\mathbf{z}}(\mathbf{x}) f(\mathbf{x}) \xi(\mathrm{d} \mathbf{x})$

$\mathbf{I}\left(\boldsymbol{\theta}_{0}\right)$ asymptotic information matrix of $\theta_{0}$

$\mathbf{C}\left(\boldsymbol{\theta}_{0}\right)$ asymptotic variance-covariance matrix of the score function evaluated at $\boldsymbol{\theta}_{0}$

$k(\mathbf{x})$ the density of $\xi(\mathbf{x})$

$\mathbf{K} \int_{S} a^{2}(\mathbf{x}) k^{2}(\mathbf{x}) \tilde{\mathbf{z}}(\mathbf{x}) \tilde{\mathbf{z}}^{\mathrm{T}}(\mathbf{x}) \mathrm{d} \mathbf{x}$

$\mathscr{F}_{1}$ contamination class for prediction problems

$\mathscr{F} 3$ contamination class for general extrapolation

$\lambda_{k}^{(1)}$ the largest solution to $\left|\mathbf{G}-\lambda \mathbf{H}_{S}\right|=0$

$\mathbf{c}_{1}$ any vector satisfying $\left(\mathbf{G H}_{S}^{-1} \mathbf{G}-\lambda_{k}^{(1)} \mathbf{G}\right) \mathbf{c}=0$, and $\mathbf{c}^{\mathrm{T}} \mathbf{G c}=1$

$\mathbf{c}_{2} \frac{\mathbf{B}^{-1} \tilde{\mathbf{z}}\left(\mathbf{x}_{0}\right)}{\sqrt{\lambda_{k}^{(2)}}}$ 
$\lambda_{k}^{(3)}$ the largest solution to $\left|\mathbf{G}-\lambda \mathbf{H}_{T}\right|=0$

$I B_{1}$ prediction bias

$I B_{3}$ general extrapolation bias

$\mathbf{G} \mathbf{K}-\mathbf{B A}_{S}^{-1} \mathbf{B}$

$\mathscr{F} 2$ contamination class for one-point extrapolation

$v \sigma^{2} / \eta_{S}^{2}$ : relative importance of variance versus bias

$\lambda_{k}^{(2)} \tilde{\mathbf{z}}^{\mathrm{T}}\left(\mathbf{x}_{0}\right) \mathbf{B}^{-1} \mathbf{G B}^{-1} \tilde{\mathbf{z}}\left(\mathbf{x}_{0}\right)$

$r_{\mathbf{x}_{0}, S} \eta_{0} / \eta_{S}$ : relative amount of response uncertainty at the extrapolation point versus within the design space

$r_{T, S} \eta_{T} / \eta_{S}$ : relative amount of response uncertainty in the extrapolation versus design spaces

$\mathbf{c}_{3}$ any vector satisfying $\left(\mathbf{G H}_{T} \mathbf{G}-\lambda_{k}^{(3)} \mathbf{G}\right) \mathbf{c}=0$

$I B_{2}$ one-point extrapolation bias

$\alpha\left\{\int_{S} a^{-1}(\mathbf{x}) \mathrm{d} \mathbf{x}\right\}^{-1}$

\section{Appendix B. Derivations}

Proof of Theorem 1. Since the term $\sigma^{2} \operatorname{tr}\left(\mathbf{A}_{S} \mathbf{B}^{-1}\right)$ in $I_{1}$ does not involve $f$, the maximization problem becomes to maximize $\mathbf{b}_{f, S}^{\mathrm{T}} \mathbf{H}_{S}^{-1} \mathbf{b}_{f, S}+\int_{S} f^{2}(\mathbf{x}) \mathrm{d} \mathbf{x}$ over $f$. We obtain

$$
\max _{f}\left(\mathbf{b}_{f, S}^{\mathrm{T}} \mathbf{H}_{S}^{-1} \mathbf{b}_{f, S}\right)=\eta_{S}^{2} \lambda_{k}^{(1)}
$$

attained at $f_{k}(\mathbf{x})=\eta_{S} \tilde{\mathbf{z}}^{\mathrm{T}}(\mathbf{x})\left\{k(\mathbf{x}) a(\mathbf{x}) \mathbf{I}-\mathbf{A}^{-1} \mathbf{B}\right\} \mathbf{c}_{1}$ in a way akin to that used in Theorem 1 of Wiens (1992), and therefore the derivations are omitted here. Theorem 1 follows immediately from $\int_{S} f_{k}^{2}(\mathbf{x}) \mathrm{d} \mathbf{x}=\eta_{S}^{2}$.

The result of Theorem 2 is obtained in a manner very similar to but simpler than that used in Theorem 1 of Wiens and Xu (2008b), and so its proof is omitted.

Theorem 3 follows Theorem 1 of Wiens and Xu (2008a) immediately.

Proof of Theorem 4. We look for a nonnegative function $k(\mathbf{x})$ minimizing (7) subject to $\int_{S} k(\mathbf{x}) \mathrm{d} \mathbf{x}=1$. We introduce a Lagrange multiplier $t$. It is sufficient to show that $k(\mathbf{x})$ minimizes

$$
\left(\sqrt{\lambda_{k}^{(2)}}+r_{\mathbf{x}_{0}, S}\right)^{2}+v \tilde{\mathbf{z}}^{\mathrm{T}}\left(\mathbf{x}_{0}\right) \mathbf{B}^{-1} \tilde{\mathbf{z}}\left(\mathbf{x}_{0}\right)-2 t \int_{S} k(\mathbf{x}) \mathrm{d} \mathbf{x}
$$

among all density functions. After some protracted calculation, we obtain the first order condition

$$
\int_{S}\{P(\mathbf{x}) k(\mathbf{x})-Q(\mathbf{x})-u\}\left(k-k_{1}\right) \mathrm{d} \mathbf{x} \geqslant 0
$$

for all densities $k_{1}$, where $P(\mathbf{x})=\left[a(\mathbf{x}) \tilde{\mathbf{z}}^{\mathrm{T}}(\mathbf{x}) \boldsymbol{\beta}\right]^{2}$ and $Q(\mathbf{x})=\left[\tilde{\mathbf{z}}^{\mathrm{T}}(\mathbf{x}) \gamma\right]\left[a(\mathbf{x}) \tilde{\mathbf{z}}^{\mathrm{T}}(\mathbf{x}) \boldsymbol{\beta}\right]$ with

$$
\begin{aligned}
& \boldsymbol{\beta}=\mathbf{B}^{-1} \tilde{\mathbf{z}}\left(\mathbf{x}_{0}\right), \\
& \gamma=\left[\mathbf{B}^{-1} \mathbf{K}+\frac{v}{2}\left(1+\frac{r_{\mathbf{x}_{0}, S}}{\sqrt{\lambda_{k}^{(2)}}}\right)^{-1} \mathbf{I}\right] \boldsymbol{\beta}, \\
& u=\left(1+\frac{r_{\mathbf{x}_{0}, S}}{\sqrt{\lambda_{k}^{(2)}}}\right)^{-1} t .
\end{aligned}
$$

The proof now can be completed in the same way as for Theorem 6 of Wiens and Xu (2008a).

Proof of Theorem 5. The term $v \operatorname{tr}\left(\mathbf{A}_{T} \mathbf{B}^{-1}\right)$ in $I_{2}$ does not involve $f$, so the maximization problem becomes maximizing $\mathbf{b}_{f, S}^{\mathrm{T}} \mathbf{H}_{T} \mathbf{b}_{f, S}$ $-2 \mathbf{b}_{f, T}^{\mathrm{T}} \mathbf{B}^{-1} \mathbf{b}_{f, S}+\int_{T} f^{2}(\mathbf{x}) \mathrm{d} \mathbf{x}$ over $f$. We obtain

$$
\max _{f}\left(\mathbf{b}_{f, S}^{\mathrm{T}} \mathbf{H}_{T} \mathbf{b}_{f, S}-2 \mathbf{b}_{f, T}^{\mathrm{T}} \mathbf{B}^{-1} \mathbf{b}_{f, S}\right)=\eta_{S}^{2}\left[\left(\sqrt{\lambda_{k}^{(3)}}+r_{T, S}\right)^{2}-r_{T, S}^{2}\right],
$$


attained at

$$
f_{k}(\mathbf{x})= \begin{cases}\eta_{S} \tilde{\mathbf{z}}^{\mathrm{T}}(\mathbf{x})\left[a(\mathbf{x}) k(\mathbf{x}) \mathbf{I}-\mathbf{A}_{S}^{-1} \mathbf{B}\right] \mathbf{c}_{3}, & \mathbf{x} \in S \\ -\eta_{T} \tilde{\mathbf{z}}^{\mathrm{T}}(\mathbf{x}) \mathbf{B}^{-1} \mathbf{G} \mathbf{c}_{3} / \sqrt{\lambda_{k}^{(3)},} & \mathbf{x} \in T,\end{cases}
$$

in a way essentially identical to that used in Theorem 2.1 (a) of Fang and Wiens (1999). Theorem 5 follows immediately from $\int_{S} f_{k}^{2}(\mathbf{x}) \mathrm{d} \mathbf{x}=\eta_{S}^{2}$.

The proof of Theorem 6 is very similar to that of Theorem 1 in Wiens and Xu (2008b), so is omitted.

Proof of Theorem 7. According to the results of Theorems 1, 3, and 5, we have got

$$
\begin{aligned}
& \sup _{f \in \mathscr{F} 1} I B_{1}(f, k)=\eta_{S}^{2} \lambda_{k}^{(1)}, \\
& \sup _{f \in \mathscr{F}_{2}} I B_{2}(f, k)=\eta_{S}^{2}\left[\left(\sqrt{\lambda_{k}^{(2)}}+r_{\mathbf{x}_{0}, S}\right)^{2}-r_{\mathbf{x}_{0}, S}^{2}\right], \\
& \sup _{f \in \mathscr{F} 3} I B_{3}(f, k)=\eta_{S}^{2}\left[\left(\sqrt{\lambda_{k}^{(3)}}+r_{T, S}\right)^{2}-r_{T, S}^{2}\right] .
\end{aligned}
$$

For the unbiased designs, we then have

$$
\begin{aligned}
\sup _{f \in \mathscr{F}_{i}} I B_{i}(f, k) & =0 \Leftrightarrow \lambda_{k}^{(i)}=0 \Leftrightarrow \mathbf{G}=\mathbf{0} \\
& \Leftrightarrow\left[a(\mathbf{x}) k(\mathbf{x}) \mathbf{I}-\mathbf{B} \mathbf{A}_{S}^{-1}\right] \mathbf{z}(\mathbf{x})=0 \quad \text { a. e. }
\end{aligned}
$$

for each $i=1,2$, or 3 . We find that $a(\mathbf{x}) k(\mathbf{x})$ is a constant almost everywhere on $S$ in a manner essentially identical to that in the proof of Theorem 2.2 (b) in Fang and Wiens (1999). This fact together with $\int_{S} k(\mathbf{x}) \mathrm{d} \mathbf{x}=1$, completes the proof of (a) and Part (b), (c) follow (a) immediately.

Proof of Theorem 8. To illustrate this, we put the $x_{i}$ in increasing order, i.e. $x_{1}<x_{2}<\ldots<x_{n}$. Let $S=[a, b]$, we then have

$$
D=\int_{a}^{x_{1}}\left|F_{\xi_{0}}(x)-F_{\xi}(x)\right|^{m} \mathrm{~d} x+\sum_{i=1}^{n-1} \int_{x_{i}}^{x_{i+1}}\left|F_{\xi_{0}}(x)-F_{\xi}(x)\right|^{m} \mathrm{~d} x+\int_{x_{n}}^{b}\left|F_{\xi_{0}}(x)-F_{\xi}(x)\right|^{m} \mathrm{~d} x .
$$

To minimize $D$, by taking the derivative with respect to each $x_{i}$, we find that the minimizing $x_{i}$ is a solution of the following equation:

$$
\left|F_{\xi_{0}}(x)-\frac{i}{n}\right|^{m}=\left|F_{\xi_{0}}(x)-\frac{i+1}{n}\right|^{m}
$$

for $i=1,2, \ldots, n$. Theorem 1 follows (11) satisfies (12) for $m>0$.

\section{References}

Adewale, A., 2002. Robust designs for approximate regression models with two interacting regressors. Unpublished Master's thesis, University of Alberta. Adewale, A., Wiens, D.P., 2006. New criteria for robust integer-valued designs in linear models. Comput. Statist. Data Anal. 51, 723-736.

Atkinson, A.C., Haines, L.M., 1996. Designs for nonlinear and generalized linear models. in: Ghosh, S., Rao, C.R. (Eds.), Handbook of Statistics, vol. 13 , pp. $437-475$. Box, G.E.P., Draper, N.R., 1959. A basis for the selection of a response surface design. J. Amer. Stat. Assoc. 54, 622-654.

Chaloner, K., Larntz, K., 1992. Bayesian design for accelerated life testing. J. Statist. Plann. Inference 33, 245-259.

Draper, N.R., Herzberg, A., 1973. Some designs for extrapolation outside a sphere. J. Roy. Statist. Soc. Ser. B 35, $268-276$

Fang, Z., 1999. Robust extrapolation designs for linear models. Unpublished Doctoral Thesis, University of Alberta.

Fang, Z., Wiens, D.P., 1999. Robust extrapolation designs and weights for biased regression models with heteroscedastic errors. Canad. J. Statist. $27,751-770$.

Fang, Z., Wiens, D.P., 2000. Integer-valued, minimax robust designs for estimation and extrapolation in heteroscedastic approximately linear models. J. Amer. Stat. Assoc. 95, 807-818.

Ford, I., Titterington, D.M., Kitsos, C.P., 1989. Recent advances in nonlinear experimental design. Technometrics 31, 49-60.

Ginebra, J., Sen, A., 1998. Minimax approach to accelerated life tests. IEEE Trans. Reliability 47, 261-267.

Heo, G., 1998. Optimal designs for approximately polynomial regression models. Unpublished Doctoral Thesis, University of Alberta.

Heo, G., Schmuland, B., Wiens, D.P., 2001. Restricted minimax robust designs for misspecified regression models. Canad. J. Statist. $29,117-128$.

Huber, P.J., 1975. Robustness and designs. In: Srivastava, J.N. (Ed.), A survey of statistical design and linear models. North-Holland, Amsterdam, pp. $287-303$.

Lawless, J.F., 1984. Some problems concerning experimental designs for extrapolation. In: Dwivedi, T.D. (Ed.), Topics in Statistics. Concordia University Press, Montreal, pp. 357-366.

Nelson, W., 1990. Accelerated Testing-Statistical Models, Test Plans, and Data Analyses. Wiley, New York.

Pascual, F.G., 2006. Accelerated life test plans robust to misspecification of the stress-life relationship. Technometrics 48, 11-25. 
Pascual, F.G., Montepiedra, G., 2002. On minimax designs when there are two candidate models. J. Statist. Comput. Simulation 72, $841-862$.

Pascual, F.G., Montepiedra, G., 2003. Model-robust test plans with applications in accelerated life testing. Technometrics 45, 47-57.

Sinha, S., Wiens, D.P., 2002. Robust sequential designs for nonlinear regression. Canad. J. Statist. 30, 601-618.

Spruill, M.C., 1984. Optimal designs for minimax extrapolation. J. Multivariate Anal. 15, 52-62.

Wiens, D.P., 1990. Robust, minimax designs for multiple linear regression. Linear Algebra Appl. 127, $327-340$ (Second Special Issue on Linear Algebra and Statistics).

Wiens, D.P., 1992. Minimax designs for approximately linear regression. J. Statist. Plann. Inference 31, 353-371.

Wiens, D.P., 1998. Minimax robust designs and weights for approximately specified regression models with heteroscedastic errors. J. Amer. Stat. Assoc. 93, $1440-1450$.

Wiens, D.P., Xu, X., 2008a. Robust designs for one-point extrapolation. J. Statist. Plann. Inference, in press.

Wiens, D.P., Xu, X., 2008b. Robust prediction and extrapolation designs for misspecified generalized linear regression models. J. Statist. Plann. Inference 138, 30-46.

Wiens, D.P., Zhou, J., 1996. Minimax regression designs for approximately linear models with autocorrelated errors. J. Statist. Plann. Inference 55, 95-106.

Zhang, Y., Meeker, W.Q., 2006. Bayesian methods for planning accelerated life test. Technometrics 48, 49-60. 